\title{
A Multidimensional Superposition Principle: Classical Solitons IV
}

Alexander A. Alexeyev

To cite this article: Alexander A. Alexeyev (2018) A Multidimensional Superposition Principle: Classical Solitons IV, Journal of Nonlinear Mathematical Physics 25:1, 1-33, DOI: https://doi.org/10.1080/14029251.2018.1440740

To link to this article: https://doi.org/10.1080/14029251.2018.1440740

Published online: 04 January 2021 


\title{
A Multidimensional Superposition Principle: Classical Solitons IV
}

\author{
Alexander A. Alexeyev \\ Department of Mathematic, Institute for Cybernetics, Moscow Technological University, \\ 78 Vernadskogo Avenue, Moscow 117454, Russian Federation \\ aalexeyev@mtu-net.ru
}

Received 8 January 2017

Accepted 14 July 2017

\begin{abstract}
This article continues the series of the works of 1998-2007 years devoted to the Multidimensional Superposition Principle, the concept easily explaining both classical soliton and more complex wave interactions in nonlinear PDEs and allowing one, in particular, to construct the general Superposition Formulae for nonlinear wave interactions. In the present research the technique of multiexpansions with constraints is considered for finding the above SFs and investigation of the related solitons. (The simplest case of such expansions technically is analogous to the so-called invariant truncated singular expansions.) As the applications, the soliton SFs of the $\mathrm{MKdV}_{ \pm}$, Kaup-Kupershmidt and new $\mathrm{A}_{ \pm}$equation are obtained for the bell-shape exponential solitons of the various families, algebraic solitons, and the configuration of the two noninteracting kinks. The linearized, parameterized versions of these SFs are investigated then, and the related analysis of the interactions is presented. The obtained results allow one to consider the one soliton solutions mentioned as the strong bound states of the simpler solitons. Concerning the results for the above concrete nonlinear PDEs, the approach being developed made it possible both to obtain the new results and to reveal new moments for the already known ones.
\end{abstract}

Keywords: Solitons; Nonlinear interactions; Superposition formulae.

2000 Mathematics Subject Classification: 35Q51, 35Q53, 37K35, 37K40

\section{Introduction}

As since the publication of the last work from the series [3]- [11] of 1998-2007 years on the Multidimensional Superposition Principle ten years have passed already, it is reasonable to recall here its main points and stages for the readers.

The MSP was born as an answer to finding of the anomalous kinks interactions with the switching effect in hypothetic electrolytes [12,13]. Since the proposed nonlinear model possessed no properties characteristic for integrable systems, there was no hope to apply all known at that moment for the last ones effective machinery for the equation derived. Moreover, the powerful switching effect without any visible inelastic consequences demonstrated in the computer simulations was new and unusual for the known soliton models. As a result, a new paradigm of soliton interactions not based on the Inverse Scattering Transform, the Hirota's method or other known approaches was needed. Such a concept, in which the existence of solitons and the possibility of the various switching effects (the simplest one obviously is the famous soliton phase shift) for their interactions were incorporated, was proposed soon, and the first results were presented on the International Mathematical Congress in 1998 year [3]. That first technique used the structures arising in the framework of the well-known truncated singular expansions (or the Painlevé expansions) approach [16,31] and 
shown that such truncated expansions are basically the very superposition formulae for solitonperturbations interactions. Starting from them, two soliton (bell-shape ones, kinks or poles) solutions were derived for several nonlinear PDEs such as the MKdV, Burgers, Sawada-Kotera and other equations. The fact that at that moment the truncated singular expansions were constructed for many integrable soliton equations allowed one to basically close the question about the SFs for their solitons and was reduced obtaining these SFs to the simple procedure with changing notations. The existence of classical elastic soliton interactions in some nonintegrable systems together with the prediction of some type inelastic interactions [6] were other important moments in the author's opinion.

In the work [8] the soliton invariant manifolds were introduced into the theory explicitly and in the full volume, that allowed one to construct the algorithmic procedure for goal seeking the nonlinear equations having solitons. The approach deals with a so-called system of the determining equations for this and demands the use of the special computer algebra software, e.g. RifSimp [32] on Maple or Crack [33] on Reduce.

The paper [10] was considering another technique for finding SFs using the expansions with respect to the basis functions associated with some differential equations systems of the polynomial type. The simplest cases with the second order polynomials are identical to the R Conte's formalism [16] of the truncated singular manifold method. The aim of the paper was to investigate also the higher order cases, and the nonstandard solitons describing by the special functions together with their SFs were presented, and the simplest nonintegrable model possessing such solitons were indicated.

The works [9] and [11] deal with the special computer approaches for the investigations of the soliton superposition nature itself, that does not have analogues in the scientific literature. For an example, they are able to numerically restore the linearized version of SFs, to visualise the excitations of the soliton structure and so on.

Touch now the aim and plan of the paper. Although already the very first works on the MSP allowed one to use the known truncated singular expansions to construct SFs, but, as was known from that time researches, not for all cases the standard one manifold technique appeared to be successful. For instance, such an expansion is not able to describe the bell-shape solitons of the MKdV and gives the SF only for the MKdV kink [3,6]. The situation with the Kaup-Kupershmidt equation is even more intriguing. While for two other integrable nonlinear PDEs of the fifth order, the higher $\mathrm{KdV}$ and Sawada-Kotera equation, usual truncated expansions can be directly transformed [3] into the full volume SFs, in the case with the KK it gives the SF for the very particular soliton case. The KK solitons are known as the anomalous solitons, which cannot be described, e.g., by the usual direct methods like the standard Hirota's substitution. The serious research $[29,30]$ was fulfilled to investigation this anomaly but nevertheless did not decided the problem completely. As we will see, in reality the clue to the puzzle is the use of the two manifolds. Moreover, the two manifolds approach with namely two identical branches is needed for the KK. This fact may seem astonishing. According to the popular viewpoint, the use of one more identical branch is absolutely usefulness. Together with the MKdV and KK a new soliton equation, the $\mathrm{A}_{ \pm}$equation, is considered. For it the noninteracting kinks configuration was discovered also.

The aim of this research, however, is not only expand and develop the basis functions expansions technique for SFs [10]. The SFs sought can be used for the analysis of soliton interactions. It is shown that for the analysis of some characteristics of such interactions the linearizations of SFs can be used. The special section of the paper is devoted namely linearized SFs. Linearized SFs also 
have their own rights however and may be as much important as the full SFs. In particular, they are interesting when investigating weak soliton interactions (e.g. in the ensemble of the solitons, so-called soliton gases or soliton lattices [17]), bounded soliton states [23,24], stability [21,22], etc.

Summarizing the contents of the paper, it should be stress out that this investigation demands very complicated algebra dealing with the systems of the differential and polynomial equations. All the algebra was fulfilled with the use of the special purpose symbolic software Crack [33] for the usual [20] and differential [27] Gröebner bases on Reduce system. So, we here are able to present only the main and short enough formulae and frequently in the compact, simplified form.

Ending, adduce the article plan. In Section 2 the theoretical questions are considered. Two separate subsections are devoted to the main points of the MSP itself, one manifold technique and its two manifolds extension with a constraint. In Section 3 the approach is applied for the $\mathrm{MKdV}_{ \pm}$, $\mathrm{KK}$ and $\mathrm{A}_{ \pm}$equations. The SFs and several soliton families are obtained for them. The case with the $\mathrm{MKdV}_{ \pm}$equations is relatively simple in the viewpoint of the complexity of the algebra, so all the underlying questions and algebra are considered in details to simplify the understanding of the similar but much more huge cases. The next section, Sections 4 , considers in details the linearization of the SFs obtained previously and uses them for the soliton interactions analysis. In doing so, the procedure of parametrization for such complicated SFs is introduced. The main results of the research are listed in Conclusion. Note that in order to simplify reading, each of the sections starts also with its own short introduction.

\section{The Main Preliminaries}

In this section the minimal theory on the MSP needed for understanding this investigation is presented (here only the simplest case corresponding to the well-known singular manifold technique are applied relevantly to the concrete nonlinear PDEs under consideration). It touches both the general and rigorous theory of the soliton invariant manifolds and multidimensional splitting and the direct technique with the truncated expansions with respect to the simplest basis functions. For more details about the MSP we would first of all recommend the paper [8].

\subsection{The MSP and invariant manifolds of the soliton type}

Let there to be some PDE, linear or nonlinear, for the simplicity of the evolution type, one dimensional and not depending explicitly on the independent variables

$$
u_{t}=E\left(u, u_{x}, u_{x x}, \ldots\right), \quad u=u(x, t) .
$$

Let also there to be another PDE

$$
u_{t_{1}}+u_{t_{2}}=E\left(u, u_{x_{1}}+u_{x_{2}}, u_{x_{1} x_{1}}+2 u_{x_{1} x_{2}}+u_{x_{2} x_{2}}, \ldots\right), \quad u=u\left(x_{1}, t_{1}, x_{2}, t_{2}\right)
$$

obtained from the former by the formal change of the differential operators

$$
\frac{\partial}{\partial x}=\frac{\partial}{\partial x_{1}}+\frac{\partial}{\partial x_{2}}, \quad \frac{\partial}{\partial t}=\frac{\partial}{\partial t_{1}}+\frac{\partial}{\partial t_{2}} .
$$

One will call the last equation the multidimensional adjoint or MD-adjoint to the first one. The projections of the solutions $u\left(x_{1}, t_{1}, x_{2}, t_{2}\right)$ for (2.2)

$$
u(x, t)=\left.u\left(x_{1}, t_{1}, x_{2}, t_{2}\right)\right|_{x_{1}=x_{2}=x, t_{1}=t_{2}=t}
$$


obviously are the solutions $u(x, t)$ for (2.1). If this second equation has an invariant manifold being described by the differential relations of the following forms

$$
\begin{aligned}
Q\left(u, u_{x_{1}}, \ldots, u_{n x_{1}}\right) & =0, \quad n \in \mathbb{N} \\
G_{j}\left(\frac{\partial}{\partial x_{2}} ; u, u_{x_{1}}, \ldots, u_{(n-1) x_{1}}\right) & =0, \quad j=\overline{1, n^{\prime}} ; n^{\prime} \in \mathbb{N},
\end{aligned}
$$

then Eq. (2.5) will be called a soliton envelope equation, Eqs. (2.6) are linkage equations, and the above invariant manifold itself is an Invariant Manifold of the Soliton type. As a consequence of (2.5) and (2.6), functions $u\left(x_{1}, t_{1}, x_{2}, t_{2}\right)$ will be of the structure

$$
u\left(x_{1}, t_{1}, x_{2}, t_{2}\right)=F\left(x_{1}, t_{1} ; \vartheta_{1}\left(x_{2}, t_{2}\right), \ldots, \vartheta_{n}\left(x_{2}, t_{2}\right)\right),
$$

with the form of $F$ is determined by the ODE (2.5), while the remaining equations, Eqs. (2.6), determine the differential linkages with respect to $x_{2}$ between $\vartheta_{j}\left(x_{2}, t_{2}\right)$ in (2.7). The solution $u\left(x_{1}, t_{1}, x_{2}, t_{2}\right)$ and respectively $u(x, t)$ in (2.4) can be interpreted as a soliton with the parameters $\vartheta_{j}$ modulated by some perturbation and describe their mutual (the soliton and a perturbation) superposition. We will say that (2.7) is the Superposition Formula for the soliton and another wave. In doing so, Eq. (2.2) leads to the split set of the equations determining the separate evolution of the soliton and perturbation.

As an example, one will shortly adduce the results $[6,8]$ for the kink-perturbation SF of the $\mathrm{MKdV}_{-}$equation

$$
u_{t}-6 u^{2} u_{x}+u_{x x x}=0, \quad u=u(x, t) .
$$

The soliton envelope equation (2.5) and linkage equations (2.6) are as follows

$$
\begin{gathered}
2 u_{x_{1} x_{1} x_{1}} u_{x_{1}}-3 u_{x_{1} x_{1}}^{2}+k^{2} u_{x_{1}}^{2}=0, \quad k=\mathrm{const}, \\
u_{x_{1} x_{1}}+u_{x_{1} x_{2}} \mp 2 u u_{x_{1}}=0,2 u_{x_{1} x_{1} x_{2}} u_{x_{1}}+3 u_{x_{1} x_{1}}^{2} \mp 4\left(u_{x_{1} x_{1}} u_{x_{1}} u+u_{x_{1}}^{3}\right)-k^{2} u_{x_{1}}^{2}=0 .
\end{gathered}
$$

So, there are two linkages to the three 'parameters' associated with the soliton envelope equation (2.8). The related $\mathrm{SF}(k \neq 0)$ is

$$
\begin{aligned}
u\left(x_{1}, t_{1}, x_{2}, t_{2}\right) & = \pm\left[\left(\frac{k+\vartheta_{x_{2}}}{2}\right) \tanh \left(\frac{k x_{1}+\frac{k^{3}}{2} t_{1}+\Delta+\vartheta}{2}\right)-\frac{\vartheta_{x_{2} x_{2}}}{2\left(k+\vartheta_{x_{2}}\right)}\right] \\
k, \Delta & =\text { const, } \vartheta=\vartheta\left(x_{2}, t_{2}\right)
\end{aligned}
$$

with $\vartheta$ satisfying the equation

$$
2 \vartheta_{t_{2}}+2 \vartheta_{x_{2} x_{2} x_{2}}-\vartheta_{x_{2}}^{3}-3 k \vartheta_{x_{2}}^{2}-3 k^{2} \vartheta_{x_{2}}-\frac{3 \vartheta_{x_{2} x_{2}}^{2}}{\vartheta_{x_{2}}+k}=0, \quad \vartheta=\vartheta\left(x_{2}, t_{2}\right)
$$

and obviously describes the interaction of the kink

$$
\left.u\left(x_{1}, t_{1}, x_{2}, t_{2}\right)\right|_{\vartheta=0}=u\left(x_{1}, t_{1}\right)=\frac{k}{2} \tanh \left(\frac{k x_{1}+\frac{k^{3}}{2} t_{1}+\Delta}{2}\right)
$$

and an arbitrary perturbation (here $k>0$ for the definiteness)

$$
\lim _{x_{1} \rightarrow \pm \infty} u\left(x_{1}, t_{1}, x_{2}, t_{2}\right)=u\left(x_{2}, t_{2}\right)= \pm\left(\frac{k+\vartheta_{x_{2}}}{2}\right)-\frac{\vartheta_{x_{2} x_{2}}}{2\left(k+\vartheta_{x_{2}}\right)} .
$$


(The expressions (2.11) and (2.12) are given for the sign '+' in (2.9).) In the simplest case $\vartheta\left( \pm \infty, t_{2}\right)=\vartheta_{ \pm \infty}=$ const this interaction leads to the kink phase shift $\left(\vartheta_{+\infty}-\vartheta_{-\infty}\right) / k$, while its effect to the perturbation is more complicated. In particular, it includes the perturbation turn-over. The more difficult case is, e.g, the capture of the kink by a perturbation [6].

\subsection{The MSP and truncated singular expansions}

As was shown already in the very first works on the multidimensional superposition principle, the well-know truncated singular expansions [31], [16] directly lead to the SFs for classical solitons. The procedure is trivial and fully straightforward [3]- [8], [10]. Let an equation under consideration

$$
E([u])=0, \quad u=u(x, t)
$$

admit of the above-mentioned expansion

$$
u(x, t)=\sum_{j=0}^{m} W_{j}([S],[C]) V(x, t)^{j}, \quad m \in \mathbb{N}
$$

with the singular manifold equation

$$
E_{\mathrm{SME}}([S],[C])=0 .
$$

The function $V(x, t)$ is associated with the singular manifold and satisfies the system

$$
V_{x}=-V^{2}-\frac{S}{2}, \quad V_{t}=C V^{2}-C_{x} V+\frac{C S+C_{x x}}{2}, \quad S=S(x, t), C=C(x, t)
$$

with the compatibility condition $V_{x t}-V_{t x}=0$ or

$$
S_{t}+C_{x x x}+2 S C_{x}+C S_{x}=0 .
$$

Here we use the formalism introduced by R Conte in the work [16]. Historically, the above series are associated with the so-called generalized Laurent expansions [31] with respect to the singular manifold function $f(x, t)$

$$
u(x, t)=\sum_{j=-m}^{+\infty} w_{j}(x, t) f^{j}(x, t), \quad m \in \mathbb{N}
$$

arose in the framework of the hypothesis on the Painlevé properties for the nonlinear PDEs integrable via the Inverse Scattering Transform [1]. Conte however shown that the series (2.17) can be presented in the more elegant and compact form (2.14) in terms of the above functions $V, S$ and $C$ which have the simple link with the singular function $f(x, t)$ from (2.17)

$$
V(x, t)=\frac{f_{x}}{f}-\frac{f_{x x}}{2 f_{x}}, \quad S(x, t)=\frac{f_{x x x}}{f_{x}}-\frac{3}{2} \frac{f_{x x}^{2}}{f_{x}^{2}}, \quad C(x, t)=-\frac{f_{t}}{f_{x}} .
$$

As was demonstrated in [10], the expansions analogous (2.14) can take place also for more complicated than the system (2.16) cases, and some special solitons together with their SFs were obtained there for already the nonintegrable models. 
If the singular manifold equation (2.15) has solutions for some constant values of $C$ and $S$, that corresponds to the existence of a soliton for the nonlinear PDE, then the SF for the last one is restored from (2.14) via the following relations

$$
\begin{aligned}
& V\left(x_{1}, t_{1}, x_{2}, t_{2}\right)=\frac{k}{2}\left(1+\theta_{x_{2}}\right) \tanh \left[\frac{k}{2}\left(x_{1}-v t_{1}+\Delta+\theta\right)\right]-\frac{\theta_{x_{2} x_{2}}}{2\left(1+\theta_{x_{2}}\right)}, \\
& S\left(x_{2}, t_{2}\right)=\frac{\theta_{x_{2} x_{2} x_{2}}}{1+\theta_{x_{2}}}-\frac{k^{2}}{2}\left(1+\theta_{x_{2}}\right)^{2}-\frac{3}{2}\left(\frac{\theta_{x_{2} x_{2}}}{1+\theta_{x_{2}}}\right)^{2}, \\
& C\left(x_{2}, t_{2}\right)=\frac{v-\theta_{t_{2}}}{1+\theta_{x_{2}}}, \quad \theta=\theta\left(x_{2}, t_{2}\right), \quad k, v, \Delta=\mathrm{const} ; k \neq 0
\end{aligned}
$$

or

$$
\begin{aligned}
& V\left(x_{1}, t_{1}, x_{2}, t_{2}\right)=\frac{1+\theta_{x_{2}}}{x_{1}-v t_{1}+\Delta+\theta}-\frac{\theta_{x_{2} x_{2}}}{2\left(1+\theta_{x_{2}}\right)}, \quad \theta=\theta\left(x_{2}, t_{2}\right) \\
& S\left(x_{2}, t_{2}\right)=\frac{\theta_{x_{2} x_{2} x_{2}}}{1+\theta_{x_{2}}}-\frac{3}{2}\left(\frac{\theta_{x_{2} x_{2}}}{1+\theta_{x_{2}}}\right)^{2}, C\left(x_{2}, t_{2}\right)=\frac{v-\theta_{t_{2}}}{1+\theta_{x_{2}}}, \quad v, \Delta=\mathrm{const}
\end{aligned}
$$

and of cause (2.3). In doing so, the singular manifold equation (2.15) brings to the governing equation for the perturbation function $\theta$. As a consequence, in view of the form of (2.14), all such SFs will be of the following standard structures [8]

$$
\begin{gathered}
u_{\mathrm{SF}, \mathrm{I}}\left(x_{1}, t_{1}, x_{2}, t_{2}\right)=\sum_{j=0}^{m} \Phi_{j}([\theta], k, v) \tanh ^{j}\left[\frac{k}{2}\left(x_{1}-v t_{1}+\Delta+\theta\right)\right], \\
u_{\mathrm{SF}, \mathrm{II}}\left(x_{1}, t_{1}, x_{2}, t_{2}\right)=\sum_{j=0}^{m} \frac{\Phi_{j}([\theta], v)}{\left[x_{1}-v t_{1}+\Delta+\theta\right]^{j}}, \quad m \in \mathbb{N}
\end{gathered}
$$

for (2.18) and (2.19) respectively, and the interactions are of the unified features. In particular, they leads to the soliton phase shifts. In their turn the expressions for the perturbations before and after the interactions have the form

$$
\begin{gathered}
u_{\mathrm{SF}, \mathrm{I}, \mathrm{p}_{ \pm \infty}}\left(x_{2}, t_{2}\right)=u_{\mathrm{SF}, \mathrm{I}}\left( \pm \infty, t_{1}, x_{2}, t_{2}\right)=\sum_{j=0}^{m}( \pm 1)^{j} \Phi_{j}([\boldsymbol{\theta}], k, v), \quad m \in \mathbb{N}, \\
u_{\mathrm{SF}, \mathrm{II}, \mathrm{p}_{ \pm \infty}}\left(x_{2}, t_{2}\right)=u_{\mathrm{SF}, \mathrm{II}}\left(\infty, t_{1}, x_{2}, t_{2}\right)=\Phi_{0}([\boldsymbol{\theta}], v) .
\end{gathered}
$$

In the case (2.22), the interaction effects the state of the perturbation after it if obviously there are odd terms. While this state in the second case (2.23) is not effected by the soliton or, say so, the soliton is transparent for perturbations.

Note that the soliton envelope equations supplying (2.20) and (2.21) can enough easily be obtained although will generally be huge enough. For instance, Eq. (2.8) is obviously the soliton envelope equation for $m=1$.

Although, as we see from the above formulae (2.18)-(2.23), there exist two qualitatively different cases of SFs, (2.20) and (2.21), but simultaneously there is the direct link between them. And all formulae for the second case with (2.21) can be formally obtained from their analogues for (2.20) simply via passage to the limit $k \rightarrow 0$. We will touch this question slightly later.

All the above facts are just as simple as important. Basically, since for the most part of the soliton equations the truncated singular expansions are already known, it closes the questions on their SFs and the nature of their soliton interactions. 
Pass now to the double expansion with the constraints. Estévez with the colleagues [18] were the first who introduced a technique with two singular manifolds. This immediately allowed one to move forward with the equations where the original one singular manifold version appeared to be powerless. A number of the interesting papers were written. And in particular the MKdV was investigated [28]. Main interest in these works was concentrated on the possibility to obtain Lax pairs, Bäcklund and Darboux transformations. In the work [14] emphasis was made on the use of constraints to the above manifolds and on the straightforward theory for finding them in the general case. As a result, for example the known problems [19] with some 'pathologic' from the classical view point equations were resolved.

The equations cases being considered in this paper are relatively simple and demand the simplest link between two singular manifold functions. And here we confine ourself to the theory only for this concrete case.

Let there to be two functions $V_{1,2}(x, t)$ satisfying as usually the systems

$$
V_{j, x}=-V_{j}^{2}-S_{j} / 2, \quad V_{j, t}=C_{j} V_{j}^{2}-C_{j, x} V_{j}+\left(C_{j} S_{j}+C_{j, x x}\right) / 2, \quad j=1,2
$$

the same as (2.16) with $S_{1,2}(x, t)$ and $C_{1,2}(x, t)$ are subject of the analogous compatibility conditions $V_{j, x t}-V_{j, t x}=0(j=1,2)$ for $(2.24)$

$$
S_{j, t}+C_{j, x x x}+2 S_{j} C_{j, x}+C_{j} S_{j, x}=0, \quad j=1,2 .
$$

The above simplest link between them is of the form

$$
L\left(V_{1}, V_{2}\right)=V_{1} V_{2}-\left(\alpha V_{1}+\beta V_{2}+\gamma-\alpha \beta\right)=0
$$

where $\alpha(x, t), \beta(x, t)$ and $\gamma(x, t)$ are undetermined yet functions. In doing so, as we will see below, their specific occurrence into (2.26) is chosen in such a manner to simplify the further expressions. In order that the link (2.26) takes place in any point $x$ and at any $t, L\left(V_{1}, V_{2}\right)$ has to be invariant with respect to differentiation on $x$ and $t$

$$
\left.L_{x}\right|_{L=0}=\left.L_{t}\right|_{L=0}=0
$$

Differentiating (2.26) on $x$, we have

$$
\begin{aligned}
-V_{1}^{2} V_{2}-V_{1} V_{2}^{2}+ & V_{1}^{2} \alpha+V_{2}^{2} \beta-V_{1}\left(\alpha_{x}+S_{2} / 2\right)-V_{2}\left(\beta_{x}+S_{1} / 2\right) \\
& +\alpha_{x} \beta+\beta_{x} \alpha-\gamma_{x}+\alpha S_{1} / 2+\beta S_{2} / 2=0
\end{aligned}
$$

or, in view of the expression for $V_{1} V_{2}$ from (2.26),

$$
\begin{gathered}
-V_{1}\left(\alpha_{x}+\alpha^{2}+\gamma+S_{2} / 2\right)-V_{2}\left(\beta_{x}+\beta^{2}+\gamma+S_{1} / 2\right)+\alpha_{x} \beta+\beta_{x} a-\gamma_{x} \\
+\alpha^{2} \beta+\alpha \beta^{2}-\alpha \gamma+\alpha S_{1} / 2-\beta \gamma+\beta S_{2} / 2=0 .
\end{gathered}
$$

Then it remains to equate the coefficients at $V_{1}, V_{2}$ and the free part in (2.27) to zero to arrive at the equations to $\alpha, \beta$ and $\gamma$

$$
\alpha_{x}=-\alpha^{2}-\gamma-S_{2} / 2, \quad \beta_{x}=-\beta^{2}-\gamma-S_{1} / 2, \quad \gamma_{x}=-2 \gamma(\alpha+\beta) .
$$


Analogously differentiating (2.26) on $t$, we will obtain other three equations

$$
\begin{aligned}
\alpha_{t} & =C_{2, x x} / 2-C_{2, x} \alpha+\alpha^{2} C_{2}+C_{1} \gamma+C_{2} S_{2} / 2, \\
\beta_{t} & =C_{1, x x} / 2-C_{1, x} \beta+\beta^{2} C_{1}+C_{1} S_{1} / 2+C_{2} \gamma \\
\gamma_{t} & =\gamma\left(-C_{1, x}-C_{2, x}+2 \alpha C_{2}+2 \beta C_{1}\right)
\end{aligned}
$$

to these functions. For (2.28), (2.29), the compatibility conditions are obviously the same (2.25) as for (2.24).

The technique of double expansions with the constraint (2.26) is similar to the usual version in the whole. Namely, we seek a solution $u(x, t)$ for an equation of interest (2.13) in the form

$$
u=W_{0}(x, t)+\sum_{j=1}^{m_{1}} W_{1, j}(x, t) V_{1}(x, t)^{j}+\sum_{j=1}^{m_{2}} W_{2, j}(x, t) V_{2}(x, t)^{j}, m_{1,2} \in \mathbb{N}
$$

without of the cross products $V_{1}^{n_{1}} V_{2}^{n_{2}}\left(n_{1} n_{2} \neq 0\right)$ in the strange of (2.26). As usually, here $m_{1,2}$ are determined by the leading terms in Eq. (2.13) and can be both different (for the different branches) and equal (in particular for the identical branches). Analogously, because of (2.26), the nonlinear PDE after the substitution (2.30) will give

$$
E_{0}+\sum_{j=1}^{M_{1}} E_{1, j} V_{1}^{j}+\sum_{j=1}^{M_{2}} E_{2, j} V_{2}^{j}=0, \quad M_{1,2} \in \mathbb{N}
$$

and after equating the coefficients $E_{0}, E_{1, j}, E_{2, j}$ in (2.31) to zero leads to the system

$$
\begin{gathered}
E_{0}\left(\left[W_{0}\right] ;\left[W_{1,1}\right], \ldots ;\left[W_{2,1}\right], \ldots ;\left[S_{1}\right],\left[C_{1}\right],\left[S_{2}\right],\left[C_{2}\right], \alpha, \beta, \gamma\right)=0, \\
E_{1, j}\left(\left[W_{0}\right] ;\left[W_{1,1}\right], \ldots ;\left[W_{2,1}\right], \ldots ;\left[S_{1}\right],\left[C_{1}\right],\left[S_{2}\right],\left[C_{2}\right], \alpha, \beta, \gamma\right)=0, \overline{j=1, M_{1}} \\
E_{2, j}\left(\left[W_{0}\right] ;\left[W_{1,1}\right], \ldots ;\left[W_{2,1}\right], \ldots ;\left[S_{1}\right],\left[C_{1}\right],\left[S_{2}\right],\left[C_{2}\right], \alpha, \beta, \gamma\right)=0, \overline{j=1, M_{2}}
\end{gathered}
$$

It is worth noting that the highest equations $E_{1, j}=0$ and $E_{2, j}=0$ for determining $W_{1, j}$ and $W_{2, j}$ in (2.33), (2.34) are of the same structure as the analogous equations in the one manifold case for the same singular branches. As a consequence, we also obtain the same expressions for $W_{1, j}, W_{2, j}$. However, the rest of the overdetermined system (2.32)-(2.34) will be much more complicated.

Introduce the splitting of the independent variables $(x, t) \rightarrow\left(x_{1}, t_{1}, x_{2}, t_{2}\right)$ and rewrite the formulae analogous to (2.18) and (2.19) $(j=1,2)$

$$
\begin{gathered}
V_{j}\left(x_{1}, t_{1},\left[\theta_{j}\right], k_{j}, v_{j}\right)=\frac{k_{j}}{2}\left(1+\theta_{j, x_{2}}\right) T_{j}-\frac{\theta_{j, x_{2} x_{2}}}{2\left(1+\theta_{j, x_{2}}\right)}, \quad \theta_{j}=\theta_{j}\left(x_{2}, t_{2}\right), \\
T_{j}\left(\eta_{j}\right)=\tanh \left(\frac{\eta_{j}}{2}\right), \eta_{j}=k_{j}\left(x_{1}-v_{j} t_{1}+\Delta_{j}+\theta_{j}\right), k_{j} \neq 0, v_{j}, \Delta_{j}=\mathrm{const}, \\
S_{j}\left(\left[\theta_{j}\right], k_{j}\right)=\frac{\theta_{j, x_{2} x_{2} x_{2}}}{1+\theta_{j, x_{2}}}-\frac{k^{2}}{2}\left(1+\theta_{j, x_{2}}\right)^{2}-\frac{3}{2}\left(\frac{\theta_{j, x_{2} x_{2}}}{1+\theta_{j, x_{2}}}\right)^{2}, C_{j}\left(\left[\theta_{j}\right], v_{j}\right)=\frac{v_{j}-\theta_{j, t_{2}}}{1+\theta_{j, x_{2}}}
\end{gathered}
$$


and

$$
\begin{aligned}
& V_{j}\left(x_{1}, t_{1},\left[\theta_{j}\right], v_{j}\right)=\left(1+\theta_{j, x_{2}}\right) P_{j}-\frac{\theta_{j, x_{2} x_{2}}}{2\left(1+\theta_{j, x_{2}}\right)}, \quad \theta_{j}=\theta_{j}\left(x_{2}, t_{2}\right), \\
& P_{j}\left(\eta_{j}\right)=\frac{1}{\eta_{j}}, \quad \eta_{j}=x_{1}-v_{j} t_{1}+\Delta_{j}+\theta_{j}, \quad v_{j}, \Delta_{j}=\mathrm{const} \\
& S_{j}\left(\left[\theta_{j}\right]\right)=\frac{\theta_{j, x_{2} x_{2} x_{2}}}{1+\theta_{j, x_{2}}}-\frac{3}{2}\left(\frac{\theta_{j, x_{2} x_{2}}}{1+\theta_{j, x_{2}}}\right)^{2}, \quad C_{j}\left(\left[\theta_{j}\right], v_{j}\right)=\frac{v_{j}-\theta_{j, t_{2}}}{1+\theta_{j, x_{2}}}
\end{aligned}
$$

respectively. After that the expressions for $\alpha, \beta, \gamma$ are trivially derived from (2.26) via the separation of the variables $\left(x_{1}, t_{1}\right)$ and $\left(x_{2}, t_{2}\right)$. We have first of all $k_{1,2}=k$ and $v_{1,2}=v$ and then

$$
\begin{aligned}
& \alpha\left(x_{2}, t_{2}\right)=\alpha\left(\theta_{1},\left[\theta_{2}\right], k\right)=-\frac{1}{2}\left\{\frac{\theta_{2, x_{2} x_{2}}}{\theta_{2, x_{2}}+1}+k\left(\theta_{2, x_{2}}+1\right) \operatorname{coth}\left[\frac{k}{2}\left(\theta_{1}-\theta_{2}\right)\right]\right\}, \\
& \beta\left(x_{2}, t_{2}\right)=\beta\left(\left[\theta_{1}\right], \theta_{2}, k\right)=-\frac{1}{2}\left\{\frac{\theta_{1, x_{2} x_{2}}}{\theta_{1, x_{2}}+1}-k\left(\theta_{1, x_{2}}+1\right) \operatorname{coth}\left[\frac{k}{2}\left(\theta_{1}-\theta_{2}\right)\right]\right\}, \\
& \gamma\left(x_{2}, t_{2}\right)=\gamma\left(\left[\theta_{1}\right],\left[\theta_{2}\right], k\right)=-\frac{k^{2}}{4} \operatorname{csch}^{2}\left[\frac{k}{2}\left(\theta_{1}-\theta_{2}\right)\right]\left(\theta_{1, x_{2}} \theta_{2, x_{2}}+\theta_{1, x_{2}}+\theta_{2, x_{2}}+1\right)
\end{aligned}
$$

for the case with (2.35), and for the case (2.36) respectively

$$
\begin{aligned}
& \alpha\left(x_{2}, t_{2}\right)=\alpha\left(\theta_{1},\left[\theta_{2}\right]\right)=-\frac{\theta_{2, x_{2} x_{2}}}{2\left(\theta_{2, x_{2}}+1\right)}-\frac{\theta_{2, x_{2}}+1}{\theta_{1}-\theta_{2}}, \\
& \beta\left(x_{2}, t_{2}\right)=\beta\left(\left[\theta_{1}\right], \theta_{2}\right)=-\frac{\theta_{1, x_{2} x_{2}}}{2\left(\theta_{1, x_{2}}+1\right)}+\frac{\theta_{1, x_{2}}+1}{\theta_{1}-\theta_{2}}, \\
& \gamma\left(x_{2}, t_{2}\right)=\gamma\left(\left[\theta_{1}\right],\left[\theta_{2}\right]\right)=-\frac{\theta_{1, x_{2}} \theta_{2, x_{2}}+\theta_{1, x_{2}}+\theta_{2, x_{2}}+1}{\left(\theta_{1}-\theta_{2}\right)^{2}} .
\end{aligned}
$$

As a result, the expansion (2.30) becomes the SF. Generally, for such a SF there will be two equations to the functions $\theta_{1,2}$ with one constraint, $u_{\mathrm{SME}, j}=0(j=\overline{1,3})$.

Note the limit expressions for the functions $T_{1,2}, V_{1,2}$ in (2.35) and $P_{1,2}, V_{1,2}$ in (2.36) associated with the states of the perturbations before and after the interactions (see (2.9) and (2.12) as the example)

$$
\begin{gathered}
T_{j}\left( \pm \infty, t_{1}, x_{2}, t_{2}\right)= \pm 1, \quad k>0, \\
V_{j}\left( \pm \infty, t_{1}, x_{2}, t_{2}\right)=V_{j}\left(x_{2}, t_{2}\right)= \pm k\left(\frac{1+\theta_{j, x_{2}}}{2}\right)-\frac{\theta_{j, x_{2} x_{2}}}{2\left(1+\theta_{j, x_{2}}\right)}, \quad j=1,2
\end{gathered}
$$

and

$$
\begin{gathered}
P_{j}\left( \pm \infty, t_{1}, x_{2}, t_{2}\right)=0, \\
V_{j}\left( \pm \infty, t_{1}, x_{2}, t_{2}\right)=V_{j}\left(x_{2}, t_{2}\right)=-\frac{\theta_{j, x_{2} x_{2}}}{2\left(1+\theta_{j, x_{2}}\right)}, \quad j=1,2 .
\end{gathered}
$$

As was mentioned before, we can directly transform the final expressions for the SF of the case (2.35) to the SF with (2.36), because there is the simple enough link between their ingredients

$$
\begin{gathered}
V_{j}\left(x_{1}, t_{1},\left[\theta_{j}\right], v\right)=\lim _{k \rightarrow 0} V\left(x_{1}, t_{1},\left[\theta_{j}\right], k, v(k)\right), \quad j=1,2 \\
S\left(\left[\theta_{j}\right]\right)=\lim _{k \rightarrow 0} S\left(\left[\theta_{j}\right], k\right), \quad C\left(\left[\theta_{j}\right], v\right)=\lim _{k \rightarrow 0} C\left(\left[\theta_{j}\right], v(k)\right),
\end{gathered}
$$


and also

$$
\begin{gathered}
\alpha\left(\theta_{1},\left[\theta_{2}\right]\right)=\lim _{k \rightarrow 0} \alpha\left(\theta_{1},\left[\theta_{2}\right], k\right), \\
\beta\left(\left[\theta_{1}\right], \theta_{2}\right)=\lim _{k \rightarrow 0} \alpha\left(\left[\theta_{1}\right], \theta_{2}, k\right), \\
\gamma\left(\left[\theta_{1}\right],\left[\theta_{2}\right]\right)=\lim _{k \rightarrow 0} \gamma\left(\left[\theta_{1}\right],\left[\theta_{2}\right], k\right),
\end{gathered}
$$

so that as a result

$$
\begin{gathered}
u_{\mathrm{SF}}\left(P_{1}, P_{2},\left[\theta_{1}\right],\left[\theta_{2}\right], v\right)=\lim _{k \rightarrow 0} u_{\mathrm{SF}}\left(T_{1}, T_{1},\left[\theta_{1}\right],\left[\theta_{2}\right], k, v(k)\right), \\
u_{\mathrm{SME}, \mathrm{j}}\left(x_{1}, t_{1},\left[\theta_{1}\right],\left[\theta_{2}\right], k\right)=\lim _{k \rightarrow 0} u_{\mathrm{SME}, \mathrm{j}}\left(\left[\theta_{1}\right],\left[\theta_{2}\right], k, v(k)\right) .
\end{gathered}
$$

To fulfil correctly the limiting process in (2.43), (2.48), it is necessary to change simultaneously the phases $\eta_{1,2} \rightarrow \eta_{1,2}+\pi i$ in $T_{1,2}$ (2.35), so that the relation

$$
T_{1,2} \rightarrow \frac{2}{k} P_{1,2}
$$

takes place.

For the SFs below it is also useful to mention the following properties of the functions $T_{1,2}$ and $P_{1,2}$ for the case of the complex arguments

$$
T_{1,2}=T_{1,2}\left(\mu_{1} \pm i \mu_{2}\right), \quad P_{1,2}=P_{1,2}\left(\mu_{1} \pm i \mu_{2}\right), \quad \mu_{1}, \mu_{2} \in \mathbb{R}
$$

(plus for the first index and minus for the second one). Namely, their following combinations are pure real value

$$
\begin{aligned}
& T_{1}+T_{2}=\frac{2 \sinh \mu_{1}}{\cosh \mu_{1}+\cos \mu_{2}}, \quad T_{1}^{2}+T_{2}^{2}=2 \frac{\sinh ^{2} \mu_{1}-\sin ^{2} \mu_{2}}{\left(\cosh \mu_{1}+\cos \mu_{2}\right)^{2}}, \\
& i\left(T_{1}-T_{2}\right)=\frac{-2 \sin \mu_{2}}{\cosh \mu_{1}+\cos \mu_{2}}, \quad i\left(T_{1}^{2}-T_{2}^{2}\right)=\frac{-4 \sinh \mu_{1} \sin \mu_{2}}{\left(\cosh \mu_{1}+\cos \mu_{2}\right)^{2}}
\end{aligned}
$$

and also

$$
\begin{aligned}
& P_{1}+P_{2}=\frac{2 \mu_{1}}{\mu_{1}^{2}+\mu_{2}^{2}}, \quad P_{1}^{2}+P_{2}^{2}=2 \frac{\mu_{1}^{2}-\mu_{2}^{2}}{\left(\mu_{1}^{2}+\mu_{2}^{2}\right)^{2}}, \\
& i\left(P_{1}-P_{2}\right)=\frac{2 \mu_{2}}{\mu_{1}^{2}+\mu_{2}^{2}}, \quad i\left(P_{1}^{2}-P_{2}^{2}\right)=\frac{4 \mu_{1} \mu_{2}}{\left(\mu_{1}^{2}+\mu_{2}^{2}\right)^{2}} .
\end{aligned}
$$

Finally, it is necessary to stress out that although, already having in the hands the above formulae, we can fulfil all the algebra in the terms of the original variables $\{x, t\}$ avoiding the use of the variables $\left\{x_{1}, t_{1}, x_{2}, t_{2}\right\}$, however the success of such a technique is based namely on the existence of this splitting. Indeed, both the equating to zero the coefficients in (2.31) and the derivation of the expressions (2.37), (2.38) for $\alpha, \beta, \gamma$ is separation of the above variables. In its turn the existence of the soliton invariant manifolds of the type (2.5), (2.6) provides the compatibility of the system (2.32)-(2.34). 


\section{The Superposition Formulae and Solitons for the $\mathrm{MKdV}_{ \pm}, \mathrm{KK}$, and $\mathrm{A}_{ \pm}$Equations}

In this section the theory and technique described above are used for finding the SFs for two wellknown nonlinear PDEs, $\mathrm{MKdV}_{ \pm}$and $\mathrm{KK}$ equations, and one new soliton equation, a so-called $\mathrm{A}_{ \pm}$ equation. These SFs serve also both the traditional exponential bell-shape solitons and the algebraic solitons (and the two noninteracting kink configuration for the last). All the solitons involved have the physical sense, i.e. are pure real value and without singularities on the real axis. The case with $\mathrm{MKdV}_{ \pm}$equation is the most simple from the viewpoint of all the derivation, and the detail exposition is presented for it, although the initial system of the overdetermined differential equations cannot be presented here anyway because of its huge size. The KK equation is famous by its anomalous solitons. The special and detail research was fulfilled to try to solve this mystery in the framework of the Hirota ideas [29], [30]. However, as one will be clear below, the rigourous theory demands the use of two identical branches regardless of the common viewpoint about their usefulness (the use of one branch leads only to the reduced SF with the soliton of the simplest structure corresponding to the special choice of the soliton parameters). The mentioned $\mathrm{A}_{ \pm}$equation is a new soliton equation, which was very recently constructed by the author using one of the approaches in the framework of the MSP itself (slightly more about it will be said below in the related subsection). It is worth noticing here also that the rigourous investigation gives three different soliton families for all the above nonlinear PDEs.

\subsection{The $M K d V_{ \pm}$equation}

The MKdV is the classical equation, and usual questions for it are well studied. In particular, it was investigated via the two singular manifold approach as well [28], although certainly other questions, not SFs, were considered in doing so. About the recent investigations of the solitons of its simplest modifications see [15]. The equation has two forms differing by the sign in front of the nonlinear term

$$
u_{t} \mp 6 u^{2} u_{x}+u_{x x x}=0, \quad u=u(x, t),
$$

which will be referred as the '-' and ' + ' equations respectively. The only difference here is that the pure real solutions for the former are the pure imaginary ones for the latter and vise versa. So, we will work with the $\mathrm{MKdV}_{-}$to avoid the explicit appearance of the complex numbers in the algebra and for the $\mathrm{MKdV}_{+}$rewriting only final formulas using the link $u_{\mathrm{MKdV}_{-}}=i u_{\mathrm{MKdV}_{+}}$everywhere this is needed. Also, the invariance at the change $u \rightarrow-u$ takes place and means the existence of opposite solutions.

First of all, recall that the balance between the dominant addends $u^{2} u_{x}$ and $u_{x x x}$ in (3.1) gives $m_{1,2}=1$ in (2.30) and simultaneously leads to the existence of two different branches. The related equations (2.33), (2.34) with the indexes $j=M_{1}, M_{2}$ give $W_{1,1}=1$ and $W_{2,1}=-1$ and respectively the expansion (2.30) of the type

$$
u(x, t)=V_{1}(x, t)-V_{2}(x, t)+W_{0}(x, t) .
$$

After that the rest of Eqs. (2.32)-(2.34) leads to the compatible system consisting of Eqs. (2.25) for $S_{1,2}$, Eqs. (2.28), (2.29) for $\alpha, \beta, \gamma$, and the equation

$$
W_{0, t}-6 W_{0}^{2} W_{0, x}+W_{0, x x x}=0
$$


for $W_{0}$, together with the additional constraints

$$
\begin{gathered}
C_{1}=S_{1}+\lambda, \quad C_{2}=S_{2}+\lambda, \quad \lambda=\text { const } \\
2 W_{0}-\alpha+\beta=0, \quad \alpha+\beta=0, \quad 6 \gamma-\lambda=0, \\
3 S_{1}-6 W_{0, x}+\lambda+6 W_{0}^{2}=0, \quad 3 S_{2}+6 W_{0, x}+\lambda+6 W_{0}^{2}=0 .
\end{gathered}
$$

This type system (2.25), (2.28), (2.29) and (3.3) with the constraints (3.4)-(3.6) is compatible, and when we transfer to the related equations to the modulation functions $\theta_{1}, \theta_{2}$ via the changes (2.35) with $k_{1,2}=k$ and (2.37) (or (2.36) and (2.38)), we have the evolution equations for them from (3.4) taking in consideration (2.35) or (2.36) and the constraint from the equation $6 \gamma-\lambda=0$ in (3.5)

$$
\begin{gathered}
\theta_{j, t}=\Theta_{j}\left(\theta_{j}, \theta_{j, x}, \theta_{j, x x}, \theta_{j, x x x} ; k, \lambda, v\right), \quad j=1,2, \\
G\left(\theta_{1}, \theta_{1, x} ; \theta_{2}, \theta_{2, x} ; k, \lambda\right)=0 .
\end{gathered}
$$

All other equations and relations appear to be the consequences of (3.7) and (3.8).

Taking into account (2.35), (2.37) or (2.36) and (2.38), the resulting expressions for the SFs (3.2) and Eqs. (3.7), (3.8) in view of the expression for $W_{0}$ in (3.5) through $\alpha, \beta$ are as follows

$$
\begin{gathered}
u_{\mathrm{I}}(x, t)=\frac{k}{2}\left[\left(\theta_{1, x}+1\right) T_{1}-\left(\theta_{2, x}+1\right) T_{2}\right] \\
-\frac{1}{4}\left\{\frac{\theta_{1, x x}}{\theta_{1, x}+1}-\frac{\theta_{2, x x}}{\theta_{2, x}+1}+k \operatorname{coth}\left[\frac{k}{2}\left(\theta_{1}-\theta_{2}\right)\right]\left(\theta_{1, x}+\theta_{2, x}+2\right)\right\}, \theta_{1,2}=\theta_{1,2}(x, t), \\
2 \theta_{j, t}-\frac{3 \theta_{j, x x}^{2}}{\theta_{j, x}+1}-k^{2} \theta_{j, x}^{3}-3 k^{2} \theta_{j, x}^{2}+\left(2 \lambda-3 k^{2}\right) \theta_{j, x}+2 \theta_{j, x x x}-k^{2}+2(\lambda-v)=0, \\
\lambda \cosh \left[k\left(\theta_{1}-\theta_{2}\right)\right]+3 k^{2}\left(\theta_{1, x} \theta_{2, x}+\theta_{1, x}+\theta_{2, x}\right)+3 k^{2}-\lambda=0,
\end{gathered}
$$

for the case (2.35) and

$$
\begin{gathered}
u_{\mathrm{II}}(x, t)=\left(\theta_{1, x}+1\right) P_{1}-\left(\theta_{2, x}+1\right) P_{2} \\
-\frac{1}{4}\left[\frac{\theta_{1, x x}}{\theta_{1, x}+1}-\frac{\theta_{2, x x}}{\theta_{2, x}+1}+\frac{2\left(\theta_{1, x}+\theta_{2, x}+2\right)}{\theta_{1}-\theta_{2}}\right], \theta_{1,2}=\theta_{1,2}(x, t), \\
2 \theta_{j, t}-\frac{3 \theta_{j, x x}^{2}}{\theta_{j, x}+1}+2 \lambda \theta_{j, x}+2 \theta_{j, x x x}+2(\lambda-v)=0, \\
6\left(\theta_{1, x} \theta_{2, x}+\theta_{1, x}+\theta_{2, x}\right)+\lambda\left(\theta_{1}-\theta_{2}\right)^{2}+6=0
\end{gathered}
$$

respectively for the case (2.36). The expressions for the perturbation before and after the interactions corresponds to the degeneration of (3.9) or (3.12) (see (2.39)-(2.42))

$$
u_{\mathrm{I}, \mathrm{p}_{ \pm \infty}}=\left.u_{\mathrm{I}}\right|_{T_{1,2}= \pm 1}, \quad u_{\mathrm{II}, \mathrm{p}_{ \pm \infty}}=\left.u_{\mathrm{II}}\right|_{P_{1,2}=0} .
$$

The last step is to reduce the SFs sought, (3.9) or (3.12), to the form possessing the separation of the soliton and perturbations, simplify the formulae, and indicate the physically relevant solutions. 
Consider the first case (3.9)-(3.11) in details. First, we will recalibrate $\theta_{1,2}$

$$
\theta_{j}(x, t) \rightarrow \frac{\operatorname{Ln} d_{j}}{k}+\theta_{j}(x, t), \quad d_{j}=\text { const }, j=1,2 .
$$

The requirement that at $\theta_{1}, \theta_{2}=0$ only the soliton remains (or in the other words there is no dependence on $x_{2}, t_{2}$ ) brings to the following expressions for $d_{1,2}, v$ and $\lambda$

$$
d_{1,2}=-2 a \pm k, \quad v=k^{2}-6 a^{2}, \quad \lambda=-6 a^{2}+\frac{3}{2} k^{2}, \quad k, a=\mathrm{const},
$$

see (3.10), (3.11). Note that here and further the new constant $a$ associated with the soliton asymptotics was introduced, i.e. $u_{\mathrm{s}}(\infty, t)=a$. Also, the parameters $d_{1,2}$ are defined accurate to some multiplier corresponding to the soliton position. With (3.16) the SF (3.9) gives the soliton sought

$$
u_{\mathrm{s}_{1}}(x, t)=\frac{2 k^{2} \mathrm{e}^{\eta}}{\left[1+(-2 a+k) \mathrm{e}^{\eta}\right]\left[1+(-2 a-k) \mathrm{e}^{\eta}\right]}+a, \quad \eta=k\left[x-\left(k^{2}-6 a^{2}\right) t+\Delta\right] .
$$

When $a \in \mathbb{R}$ and $d_{1,2}$ are suitable, (3.17) is the real value, nonsingular soliton of the MKdV_. To obtain the analogous soliton of the $\mathrm{MKdV}_{+}$equation, it is enough to pass to the imaginary $a$ in (3.17). After the change $a \rightarrow i a, \mathrm{e}^{\eta} \rightarrow i \mathrm{e}^{\eta}$ (i.e. the phase shift), and $u \rightarrow i u$, one will have

$$
u_{\mathrm{s}_{2}}(x, t)=\frac{2 k^{2} \mathrm{e}^{\eta}}{\left(1+2 a \mathrm{e}^{\eta}\right)^{2}+k^{2} \mathrm{e}^{2 \eta}}+a, \quad \eta=k\left[x-\left(k^{2}+6 a^{2}\right) t+\Delta\right] .
$$

For (3.12)-(3.14) the analogous procedure with the slightly other recalibration

$$
\theta_{j}(x, t) \rightarrow d_{j}+\theta_{j}(x, t),
$$

gives (here $d_{1,2}$ are define up to already an additive constant)

$$
d_{1,2}=\mp \frac{1}{2 a}, \quad v=-6 a^{2}, \quad \lambda=-6 a^{2}, \quad a=\text { const },
$$

and (3.12) with (3.19), (3.20) brings us (also after change $a \rightarrow i a$ and $u \rightarrow i u$ as it was for (3.18)) to the real value nonsingular algebraic soliton of the $\mathrm{MKdV}_{+}$

$$
u_{\mathrm{s}_{3}}(x, t)=\frac{-4 a}{4 a^{2} \eta^{2}+1}+a, \quad \eta=x-6 a^{2} t+\Delta .
$$

For $\mathrm{MKdV}_{-}$the real value algebraic soliton also exists but has the singularities on the real axis. The expression (3.21) can also be obtained from (3.18) as the limit at $k \rightarrow 0$ with the suitable phase shift, see (2.50).

Figures 1-3 demonstrate the typical profiles of the solitons obtained. As can be seen, the exponential solitons of the first family (3.17) degenerate to the system of two noninteracting kinks (Figure 1). In other words, these solitons can be interpreted also as the strong bound kink states, not as the simple alone soliton. Figure 2 corresponds to the soliton family (3.18), while Figure 3 corresponds to the algebraic solitons (3.21).

The solutions (3.18) and (3.21) by themselves are known. They can be found, e.g., in the book [2]. While to our knowledge the strong bound state of the kinks (3.17) for the $\mathrm{MKdV}_{-}$has not attracted the attention of researches. Using the SFs found, two-soliton solutions of the various 
A.A. Alexeyev / A multidimensional superposition principle: IV

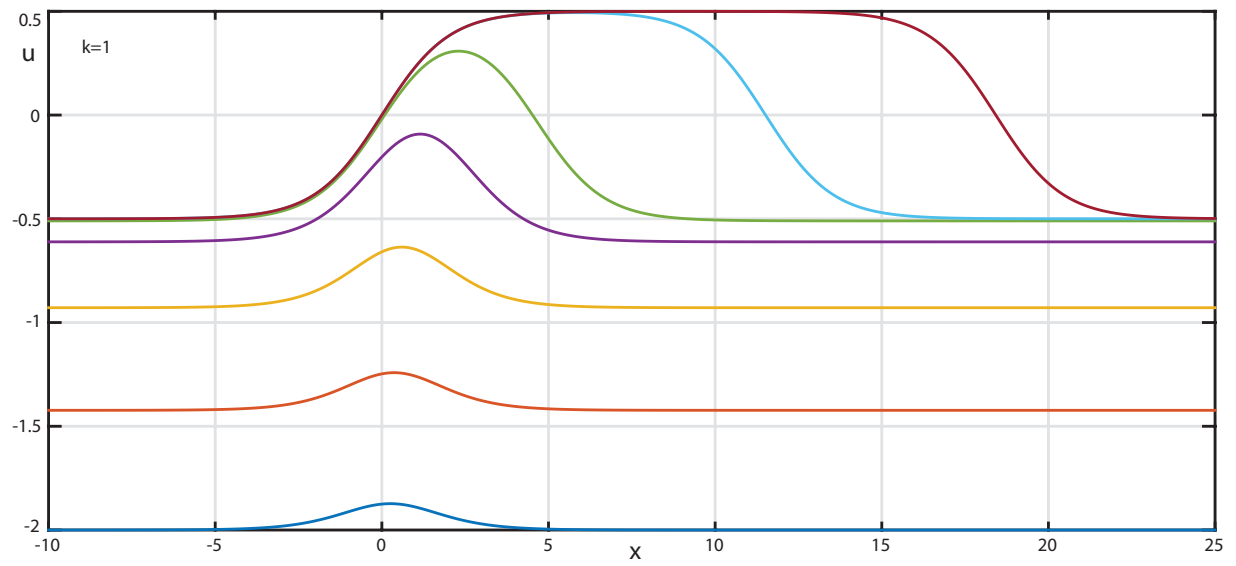

Fig. 1. The typical profiles of the soliton family (3.17) of the MKdV_ equation.

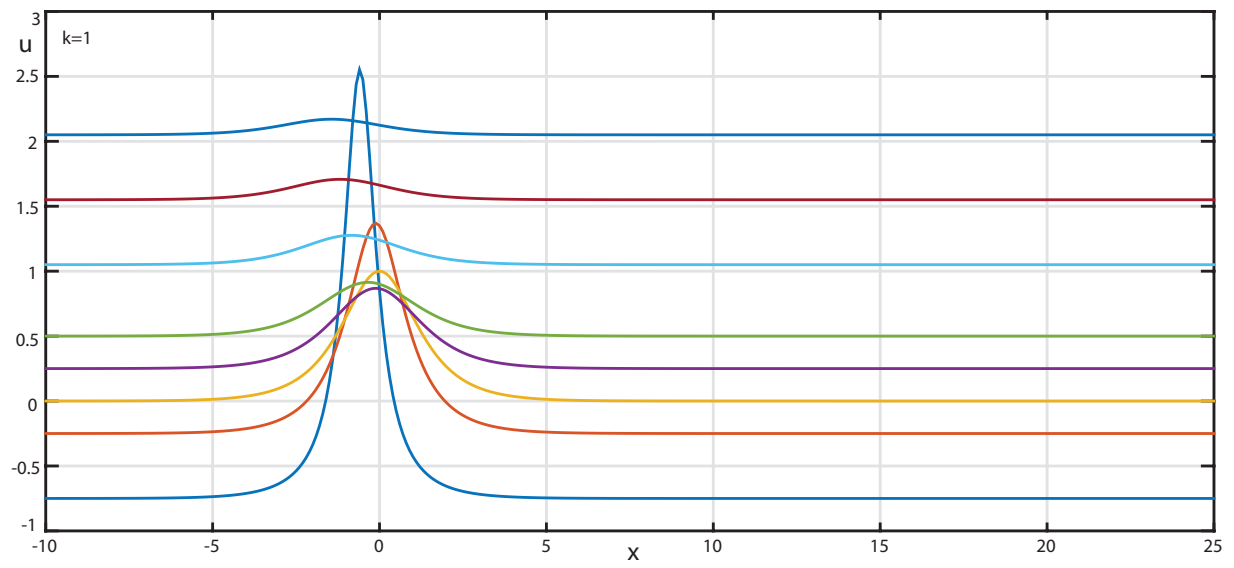

Fig. 2. The typical profiles of the soliton family (3.18) of the $\mathrm{MKdV}_{+}$equation.

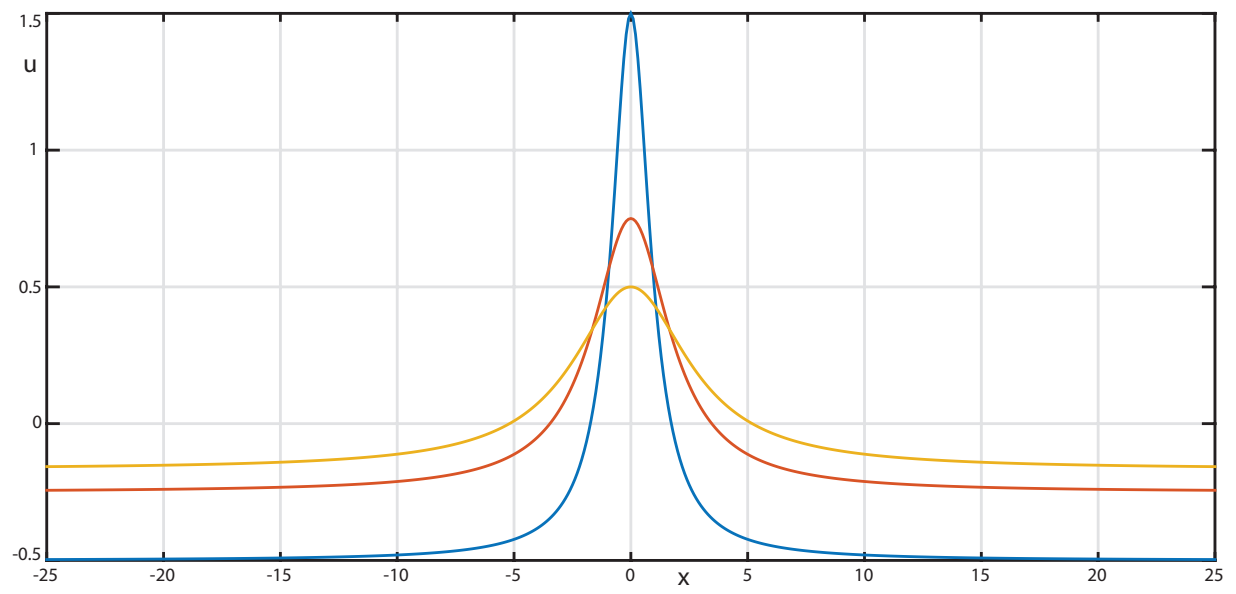

Fig. 3. The typical profiles of the algebraic solitons (3.21) of the $\mathrm{MKdV}_{+}$equation. 




Fig. 4. The interaction, see (3.22), between the pure kink solution (2.11) (the mark $A, k_{1}=1$ ) and the strong bound state of the kinks (3.17) (the mark $B, k_{2}=1-10^{-8}$ ) of the $\mathrm{MKdV}_{-}$. The coordinate system is moving together with the kink.

mixture types can be obtained. In particular, the solution describing the interaction between the kink (2.11) and the just mentioned two-kink strong bounded state (3.17) is as follows

$$
\begin{gathered}
u_{\text {interaction, } \operatorname{MKdV}_{-}}=\frac{\partial}{\partial x} \ln \left(\frac{1+E_{2}}{1+v E_{2}}+\frac{1+v^{2} E_{2}}{v+v^{2} E_{2}} E_{1}\right)-\frac{k_{1}}{2}, \quad v=\frac{k_{1}-k_{2}}{k_{1}+k_{2}} \\
E_{1}=\mathrm{e}^{k_{1} x+\frac{k_{1}^{3}}{2} t+\Delta_{1}}, E_{2}=\mathrm{e}^{k_{2} x+\left(\frac{3}{2} k_{1}^{2} k_{2}-k_{2}^{3}\right) t+\Delta_{2}} .
\end{gathered}
$$

The interaction is simple and without any resonance despite the very close wave numbers and duration, see Figure 4. In our opinion, at the present moment, obtaining of such two-solitons solutions in itself is not so interesting and important as the analysis of the SFs and general character of the interactions.

\subsection{The KK equation}

The KK equation has the following form

$$
u_{t}+180 u^{2} u_{x}+75 u_{x} u_{x x}+30 u u_{x x x}+u_{x x x x x}=0, \quad u=u(x, t)
$$

and is known no less then the MKdV. In order to obtain the full SF for its solitons, it is necessary to use the expansion (2.30) with two identical branches where $m_{1,2}=2$. As a result, the highest equations (2.33), (2.34) immediately give the simple expressions $W_{1,2}=W_{2,2}=-1 / 2$ and $W_{1,1}=$ $W_{2,1}=0$, so that (2.30) will be

$$
u(x, t)=-\frac{1}{2} V_{1}^{2}(x, t)-\frac{1}{2} V_{2}^{2}(x, t)+W_{0}(x, t) .
$$

After that, starting from the remaining equations in (2.32)-(2.34), one arrives at the compatible systems consisting of Eqs. (2.25) for $S_{1,2}$, Eqs. (2.28), (2.29) for $\alpha, \beta, \gamma$ together with the new 
equation for $W_{0}$ specific namely for (3.23)

$$
\begin{gathered}
8 W_{0, t}+8 W_{0, x x x x x}-480 W_{0, x x x} W_{0}+180 W_{0, x x x}\left(\alpha^{2}+\beta^{2}\right)-840 W_{0, x x} W_{0, x} \\
+3240 W_{0, x x} W_{0}(\alpha+\beta)+180 W_{0, x x}\left(-7 \alpha^{3}-8 \alpha^{2} \beta-8 \alpha \beta^{2}-2 \alpha \gamma-7 \beta^{3}-2 \beta \gamma\right) \\
+2160 W_{0, x}^{2}(\alpha+\beta)+31680 W_{0, x} W_{0}^{2}+4320 W_{0, x} W_{0}\left(-9 \alpha^{2}-7 \alpha \beta-9 \beta^{2}-\gamma\right) \\
+90 W_{0, x}\left(111 \alpha^{4}+140 \alpha^{3} \beta+254 \alpha^{2} \beta^{2}+52 \alpha^{2} \gamma+140 \alpha \beta^{3}+80 \alpha \beta \gamma+111 \beta^{4}\right. \\
\left.+52 \beta^{2} \gamma-16 \gamma^{2}\right)-97200 W_{0}^{3}(\alpha+\beta)+3240 W_{0}^{2}\left(41 \alpha^{3}+60 \alpha^{2} \beta+60 \alpha \beta^{2}+12 \alpha \gamma\right. \\
\left.+41 \beta^{3}+12 \beta \gamma\right)+270 W_{0}\left(-215 \alpha^{5}-403 \alpha^{4} \beta-652 \alpha^{3} \beta^{2}-158 \alpha^{3} \gamma-652 \alpha^{2} \beta^{3}\right. \\
\left.-290 \alpha^{2} \beta \gamma-403 \alpha \beta^{4}-290 \alpha \beta^{2} \gamma+56 \alpha \gamma^{2}-215 \beta^{5}-158 \beta^{3} \gamma+56 \beta \gamma^{2}\right) \\
+45\left(183 \alpha^{7}+412 \alpha^{6} \beta+856 \alpha^{5} \beta^{2}+236 \alpha^{5} \gamma+1099 \alpha^{4} \beta^{3}+578 \alpha^{4} \beta \gamma+1099 \alpha^{3} \beta^{4}\right. \\
+890 \alpha^{3} \beta^{2} \gamma-156 \alpha^{3} \gamma^{2}+856 \alpha^{2} \beta^{5}+890 \alpha^{2} \beta^{3} \gamma-172 \alpha^{2} \beta \gamma^{2}+412 \alpha \beta^{6}+578 \alpha \beta^{4} \gamma \\
\left.-172 \alpha \beta^{2} \gamma^{2}-128 \alpha \gamma^{3}+183 \beta^{7}+236 \beta^{5} \gamma-156 \beta^{3} \gamma^{2}-128 \beta \gamma^{3}\right)=0
\end{gathered}
$$

completed by two additional constraints

$$
\begin{gathered}
S_{1}-S_{2}-3\left(\alpha^{2}-\beta^{2}\right)=0, \\
12 W_{0}+S_{1}+S_{2}-3\left(\alpha^{2}+\beta^{2}\right)=0 .
\end{gathered}
$$

Besides, the following expressions for $C_{1}$ and $C_{2}$ take place

$$
\begin{gathered}
C_{1}=-6 W_{0, x x}+18 W_{0, x} \alpha+63 W_{0}^{2}+27 W_{0}\left(-3 \alpha^{2}-2 \alpha \beta-2 \beta^{2}+2 \gamma\right) \\
+\frac{81}{4} \alpha^{4}+27 \alpha^{3} \beta+36 \alpha^{2} \beta^{2}-54 \alpha^{2} \gamma+18 \alpha \beta^{3}+\frac{27}{2} \beta^{4}-27 \beta^{2} \gamma-9 \gamma^{2}, \\
C_{2}=-6 W_{0, x x}+18 W_{0, x} \beta+63 W_{0}^{2}+27 W_{0}\left(-2 \alpha^{2}-2 \alpha \beta-3 \beta^{2}+2 \gamma\right) \\
+\frac{27}{2} \alpha^{4}+18 \alpha^{3} \beta+36 \alpha^{2} \beta^{2}-27 \alpha^{2} \gamma+27 \alpha \beta^{3}+\frac{81}{4} \beta^{4}-54 \beta^{2} \gamma-9 \gamma^{2} .
\end{gathered}
$$

Just as in the case of the MKdV before, this system will give two evolution equations to the modulation functions $\theta_{1,2}$ with the only link between them

$$
\begin{gathered}
\theta_{j, t}=\Theta_{j}\left(\theta_{1}, \theta_{1, x}, \cdots, \theta_{1, x x x x x} ; \theta_{2}, \theta_{2, x}, \cdots, \theta_{2, x x x x x} ; k, v\right), \quad j=1,2, \\
G\left(\theta_{1}, \theta_{1, x}, \theta_{1, x x}, \theta_{1, x x x} ; \theta_{2}, \theta_{2, x}, \theta_{2, x x}, \theta_{2, x x x} ; k\right)=0 .
\end{gathered}
$$

(see (2.35) with $k_{1,2}=k$ and (2.37) or (2.36) and (2.38)). Firsts of them, (3.29), are obtained from (3.27), (3.28) with the expression for $W_{0}$ from (3.26) taking in consideration (2.35) or (2.36). While (3.30) is obtained from (3.25). Other equations appear to be just their consequences. Unfortunately, in the case of the KK equation the full form of Eqs. (3.29), (3.30) cannot be presented in the article because of their size (see, however, their degenerate cases in the next section). For example, Eq. (3.29) consists from 3767 terms, and (3.30) does from 144. Analogously, we can here adduce only the form for the SF (3.24) in terms of the functions $V_{1,2}, S_{1,2}, \alpha, \beta$ from (2.35), (2.37) or (2.36), (2.38)

$$
u(x, t)=-\frac{1}{2} V_{1}^{2}+\frac{1}{2} V_{2}^{2}-\frac{1}{12}\left(S_{1}+S_{2}\right)+\frac{1}{4}\left(\alpha^{2}+\beta^{2}\right) .
$$

Now we need to obtain the expression for $v$ and analyse what types of the solitons one deal with. To do this, for the usual exponential solitons one will recalibrate $\theta_{1}$ and $\theta_{2}$ in (3.29), (3.30) in the 
same manner (3.15) as previously for the MKdV. For $d_{1,2}$ and $v$ Eqs. (3.29), (3.30) at $\theta_{1}, \theta_{2}=0$, i.e. when only the soliton without a perturbation exists, give

$$
d_{1,2}=1 \pm s, \quad s^{2}=\frac{3 k^{2}}{4\left(k^{2}+6 a\right)}, \quad v=k^{4}+30 a k^{2}+180 a^{2}, \quad a=\text { const. }
$$

The related expression for the soliton is

$$
\begin{gathered}
u_{\mathrm{s}_{1}}(x, t)= \\
k^{2} \frac{\left(1-s^{2}\right) \mathrm{e}^{3 \eta}+2\left(1-s^{2}\right) \mathrm{e}^{2 \eta}+\mathrm{e}^{\eta}}{\left[1+(1+s) \mathrm{e}^{\eta}\right]^{2}\left[1+(1-s) \mathrm{e}^{\eta}\right]^{2}}+a, \quad s^{2}=\frac{3 k^{2}}{4\left(k^{2}+6 a\right)}>0, \\
\eta=k\left[x-\left(k^{4}+30 a k^{2}+180 a^{2}\right) t+\Delta\right] .
\end{gathered}
$$

Here the real, nonsingular solution will be obviously if, first, $s$ is real (i.e. $s^{2}>0$ or $a>-k^{2} / 6$ and suitable $d_{1,2}$, see (3.32)). But not only. If $s$ is pure imaginary $\left(s^{2}<0\right.$ or $\left.a<-k^{2} / 6\right)$, then expression (3.33) can also be rewritten as

$$
\begin{gathered}
u_{\mathrm{s}_{2}}(x, t)=k^{2} \frac{\left(1+\hat{s}^{2}\right) \mathrm{e}^{3 \eta}+2\left(1+\hat{s}^{2}\right) \mathrm{e}^{2 \eta}+\mathrm{e}^{\eta}}{\left[\left(1+\mathrm{e}^{\eta}\right)^{2}+\hat{s}^{2} \mathrm{e}^{2 \eta}\right]^{2}}+a, \quad \hat{s}^{2}=\frac{-3 k^{2}}{4\left(k^{2}+6 a\right)}>0, \\
\eta=k\left[x-\left(k^{4}+30 a k^{2}+180 a^{2}\right) t+\Delta\right],
\end{gathered}
$$

and this will also be the real, nonsingular solution. The real algebraic soliton in its tern can directly be obtained based on the SF (3.31) with (2.36) using the recalibration (3.19), in this case

$$
d_{1,2}= \pm d, \quad d^{2}=\frac{1}{8 a}, \quad v=180 a^{2}
$$

so as a result

$$
u_{\mathrm{S}_{3}}(x, t)=-8 a \frac{\left(1+8 a \eta^{2}\right)}{\left(1-8 a \eta^{2}\right)^{2}}+a, \quad \eta=x-180 a^{2} t+\Delta,
$$

or again via passing to the limit $k \rightarrow 0$ in (3.34). Here $a<0$ for the nonsingular case.

Figures 5-7 show the typical profiles of all the above soliton families, (3.33), (3.34) and (3.36). Again, (3.33) can be considered as the strong bound state of the simpler solitons. To the best of our knowledge the full and exhaustive researches of all three families and all the cases of these solitons (or the above soliton bound states) are not presented in the literature. Using the SFs, the interactions between the different families can in particular be investigated.

\subsection{The $A_{ \pm}$equation}

As was demonstrated in [8], the one of the powerful features of the MSP along with the transparency of the phenomenon of soliton interactions themselves is the possibility to find new nonlinear equations with a prescribed SF structure. The following equation

$$
\left(u^{3}\right)_{t}-2\left(u^{3}\right)_{x x x}+3 u_{x}\left(18 u_{x x} u+u_{x}^{2} \pm 3 p^{2} \pm 3 u^{4}\right), \quad u=u(x, t), p=\text { const }
$$

was very recently artificially constructed by the author setting the existence of the MKdV type SFs for it as the initial requirement. Its preliminary investigation (it is planing to report the details and 
A.A. Alexeyev / A multidimensional superposition principle: IV

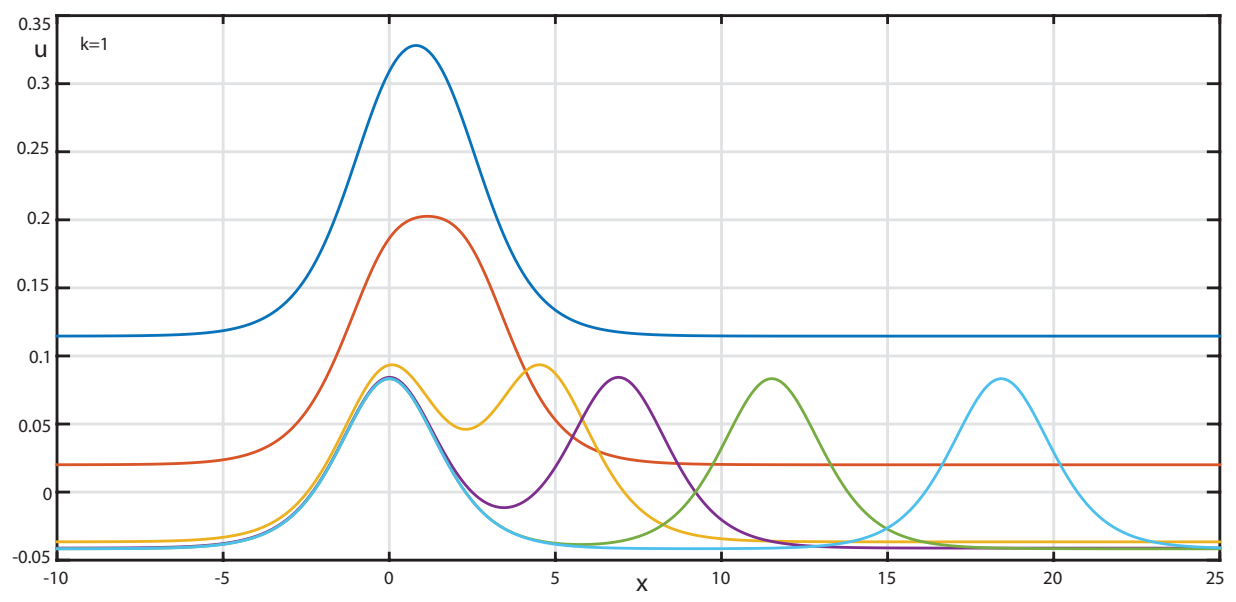

Fig. 5. The typical profiles of the soliton family (3.33) of the KK equation.

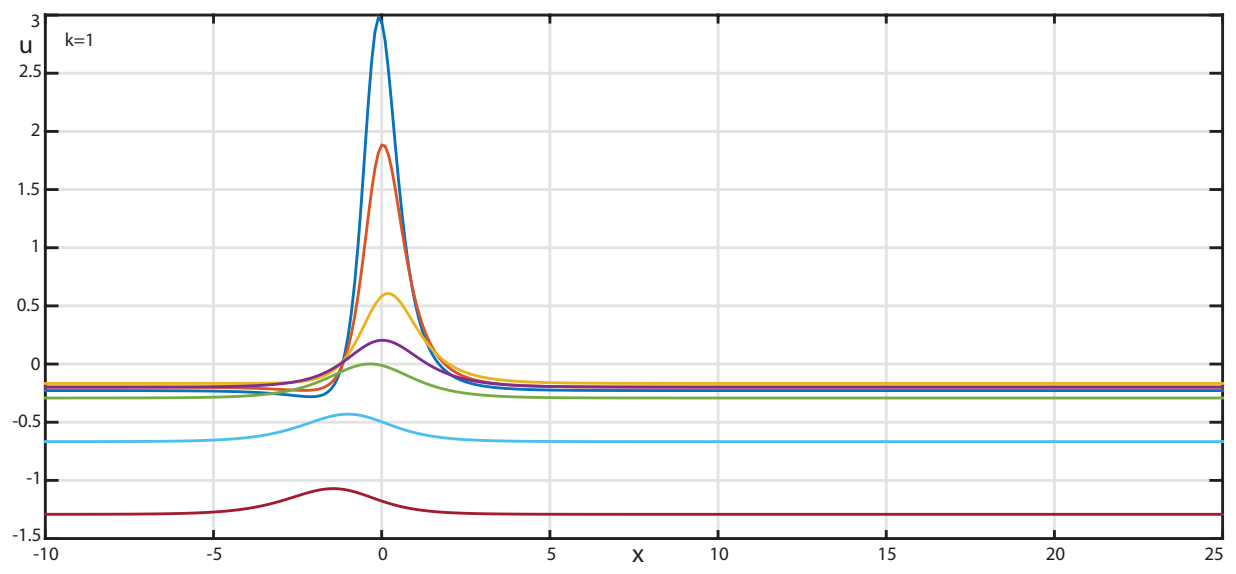

Fig. 6. The typical profiles of the soliton family (3.34) of the KK equation.



Fig. 7. The typical profiles of the algebraic solitons (3.36) of the KK equation.

Co-published by Atlantis Press and Taylor \& Francis Copyright: the authors 
technique in a separate article) shows that it accordingly possesses many solutions (the kinks, bellshape $N$-solitons and breathers) analogously to the $\mathrm{MKdV}$, but these classes are more reach. It is interesting to note here that for its degeneration with $p=0$ (this parameter is not trivial, that is reflected in particular in the SF structures and solutions, see below, without the loss of generality it can be set $p^{2}=0, \pm 1$ via scaling) directly linked with Eq. (2.10) via the transform $\vartheta_{x}=u-k$. In the paper we tentatively reference to (3.37) as to the $\mathrm{A}_{ \pm}$equation just because it was the first equation among the sought ones. As well as the MKdV, it exists in two real value versions with the ' \pm ' signs connected via the substitution $u \rightarrow i u$ and is invariant with respect to $u \rightarrow-u$. The algebra will be presented for the $\mathrm{A}_{+}$version.

From the leading equations in (2.33), (2.34), one find that there are two opposite branches with $W_{1,1}=1$ and $W_{2,1}=-1$, and the expansion (2.30) is of the form

$$
u(x, t)=V_{1}(x, t)-V_{2}(x, t)+W_{0}(x, t)
$$

The system of the remaining equations (2.32)-(2.34) is however not so simple. And not only because it is huge enough but also because it appears there are two dramatically deferent cases. As the one solution the following system in the involution takes place: the evolution equations (2.25) for $S_{1}$ and $S_{2}$ with

$$
C_{1}=\frac{1}{2}\left(12 W_{0}^{2}-24 \alpha W_{0}-S_{1}-3 S_{2}\right), C_{2}=\frac{1}{2}\left(12 W_{0}^{2}+24 \beta W_{0}-3 S_{1}-S_{2}\right),
$$

the pair of the equations to $W_{0}$

$$
\begin{aligned}
& W_{0 x}=\frac{S_{1}-S_{2}}{4}, \quad W_{0 t}=W_{0}\left(-S_{1 x}-S_{2 x}+2 \alpha^{2} W_{0}+\alpha S_{1}+\alpha S_{2}+\right. \\
& \left.2 \alpha W_{0}^{2}-2 \beta^{2} W_{0}+\beta S_{1}+\beta S_{2}+2 \beta W_{0}^{2}-2 S_{1} W_{0}+2 S_{2} W_{0}\right)
\end{aligned}
$$

(remember about the pairs (2.28), (2.29) for $\alpha, \beta, \gamma)$ plus two constraints

$$
S_{1}-S_{2}-4 W_{0}(\alpha+\beta)=0, W_{0}^{4}+2 W_{0}^{3}(\beta-\alpha)+W_{0}^{2}\left[(\alpha-\beta)^{2}+4 \gamma\right]-p^{2}=0 .
$$

Basically, the last ones give the expressions for $W_{0}$ and $\gamma$. In particular, excluding $W_{0}$ from the former, we will obtain the expression for $u(x, t)(3.38)$

$$
u(x, t)=V_{1}-V_{2}+\frac{S_{1}-S_{2}}{4(\alpha+\beta)}
$$

with the constraint (the latter)

$$
\begin{gathered}
\left(S_{1}-S_{2}\right)^{4}+8\left(S_{1}-S_{2}\right)^{3}\left(\beta^{2}-\alpha^{2}\right)+16\left(S_{1}-S_{2}\right)^{2}\left[(\alpha-\beta)^{2}+4 \gamma\right](\alpha+\beta)^{2} \\
-256 p^{2}(\alpha+\beta)^{4}=0 .
\end{gathered}
$$

In other words, now it is possible to proceed to the equations and expressions with respect to the phase functions $\theta_{1}$ and $\theta_{1}$, because all available functions, namely $S_{1,2}, C_{1,2}, \alpha, \beta, \gamma$ are their expressions (see (2.35) with $k_{1,2}=k$ and (2.37) or (2.36) and (2.38)). As a result, we will have only 
two evolution equations from (3.39) with the one constraint (3.43)

$$
\begin{gathered}
\theta_{j, t}=\Theta_{j}\left(\theta_{1}, \theta_{1, x}, \theta_{1, x x}, \theta_{1, x x x} ; \theta_{2}, \theta_{2, x}, \theta_{2, x x}, \theta_{2, x x x} ; k, v ; p\right), \quad j=1,2, \\
G\left(\theta_{1}, \theta_{1, x}, \theta_{1, x x}, \theta_{1, x x x} ; \theta_{2}, \theta_{2, x}, \theta_{2, x x}, \theta_{2, x x x} ; k ; p\right)=0
\end{gathered}
$$

(Eqs. (2.28), (2.29) and (3.40) are the consequences), and the SF (3.42) is of the form

$$
u(x, t)=U\left(T_{1}, T_{2} ; \theta_{1}, \theta_{1, x}, \theta_{1, x x}, \theta_{1, x x x} ; \theta_{2}, \theta_{2, x}, \theta_{2, x x}, \theta_{2, x x x} ; k\right) .
$$

What soliton solutions do they associated with? Again, let us introduce the recalibration (3.15) and set $\theta_{1,2}=0$ to take away any perturbations. The system (3.44), (3.45) gives the expressions for the unknown $d_{1,2}$ and the dispersion relations $v=v(k)$

$$
d_{1,2}=a^{4}+a^{2} k^{2}-p^{2} \mp 2 a^{3} k, \quad v=-2 k^{2}+3 \frac{\left(p^{2}+a^{4}\right)}{a^{2}}
$$

After that the SF (3.42) gives the clear soliton solution

$$
\begin{gathered}
u_{\mathrm{s}_{1}}(x, t)=\frac{-4 k^{2} a^{3} \mathrm{e}^{\eta}}{\left[1+\left(a^{4}+a^{2} k^{2}-p^{2}-2 a^{3} k\right) \mathrm{e}^{\eta}\right]\left[1+\left(a^{4}+a^{2} k^{2}-p^{2}+2 a^{3} k\right) \mathrm{e}^{\eta}\right]}+a, \\
\eta=k\left\{x-\left[-2 k^{2}+3 \frac{\left(p^{2}+a^{4}\right)}{a^{2}}\right] t+\Delta\right\} .
\end{gathered}
$$

This solution is the analogue of the solution (3.17). For real $a$ and suitable $d_{1,2}$ in (3.47) this is the real, nonsingular soliton of the $\mathrm{A}_{+}$. But setting $a \rightarrow i a$ and $u \rightarrow i u$, we will obtain real nonsingular soliton of the $\mathrm{A}_{-}$

$$
\begin{gathered}
u_{\mathrm{s}_{2}}(x, t)=\frac{4 k^{2} a^{3} \mathrm{e}^{\eta}}{\left[1+\left(a^{4}-a^{2} k^{2}-p^{2}\right) \mathrm{e}^{\eta}\right]^{2}+4 a^{6} k^{2} \mathrm{e}^{2 \eta}}+a, \\
\eta=k\left\{x-\left[-2 k^{2}-3 \frac{\left(p^{2}+a^{4}\right)}{a^{2}}\right] t+\Delta\right\}
\end{gathered}
$$

For the algebraic soliton with the recalibration (3.19) in (2.36), Eqs. (3.44), (3.45) give

$$
d_{1,2}= \pm \frac{2 a^{3}}{\left(p^{2}-a^{4}\right)}, \quad v=-3 \frac{\left(a^{4}+p^{2}\right)}{a^{2}} .
$$

So, finally after the change $a \rightarrow i a$ and $u \rightarrow i u$ we have the real nonsingular algebraic soliton of the $\mathrm{A}_{-}$

$$
u_{\mathrm{S}_{3}}(x, t)=4 a^{3} \frac{\left(p^{2}-a^{4}\right)}{\left(p^{2}-a^{4}\right)^{2} \eta^{2}+4 a^{6}}+a, \quad \eta=x+3 \frac{\left(a^{4}+p^{2}\right)}{a^{2}} t+\Delta .
$$

Figures $8-10$ depicts the typical profiles of the above solitons.

Now recall that the system (2.32)-(2.34) with (3.38) for Eq. (3.37) has another solution different from (3.39)-(3.41). This second solution may seem paradoxical. It shows that if $W_{0}=0, \alpha, \beta$ and $\gamma$ can be arbitrary! This means that introduction of the link (2.26) between $V_{1}$ and $V_{2}$ is not essential. After substitution (3.38) into our Eq. (3.37) we can immediately equate the coefficients 
A.A. Alexeyev / A multidimensional superposition principle: IV

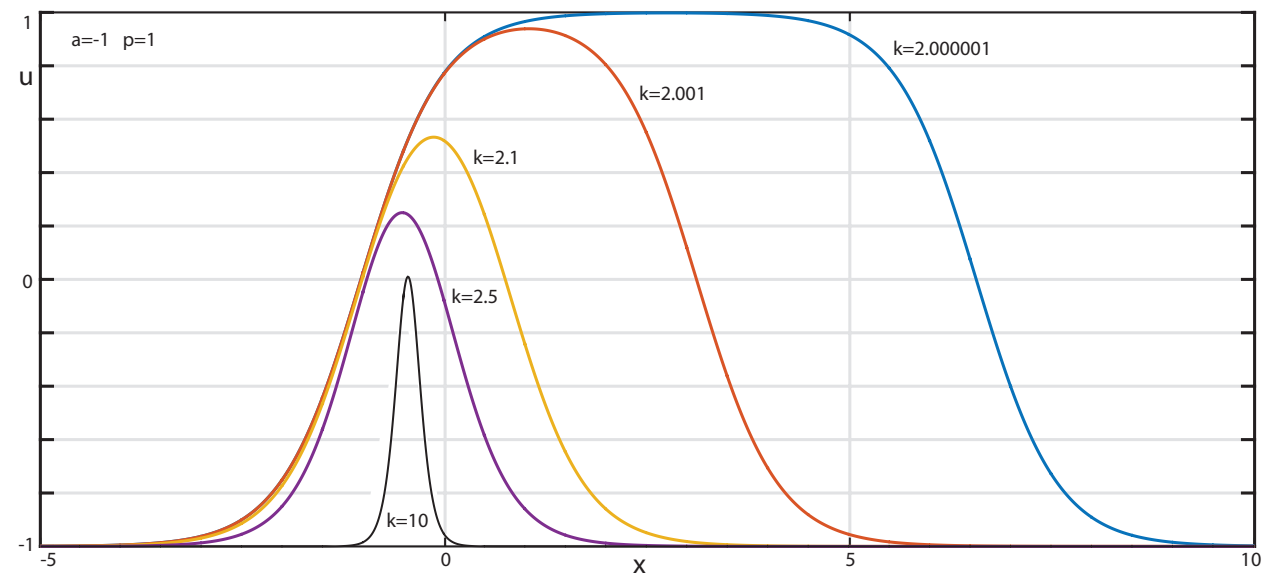

Fig. 8. The typical profiles of the soliton family (3.48) of the $\mathrm{A}_{+}$equation.

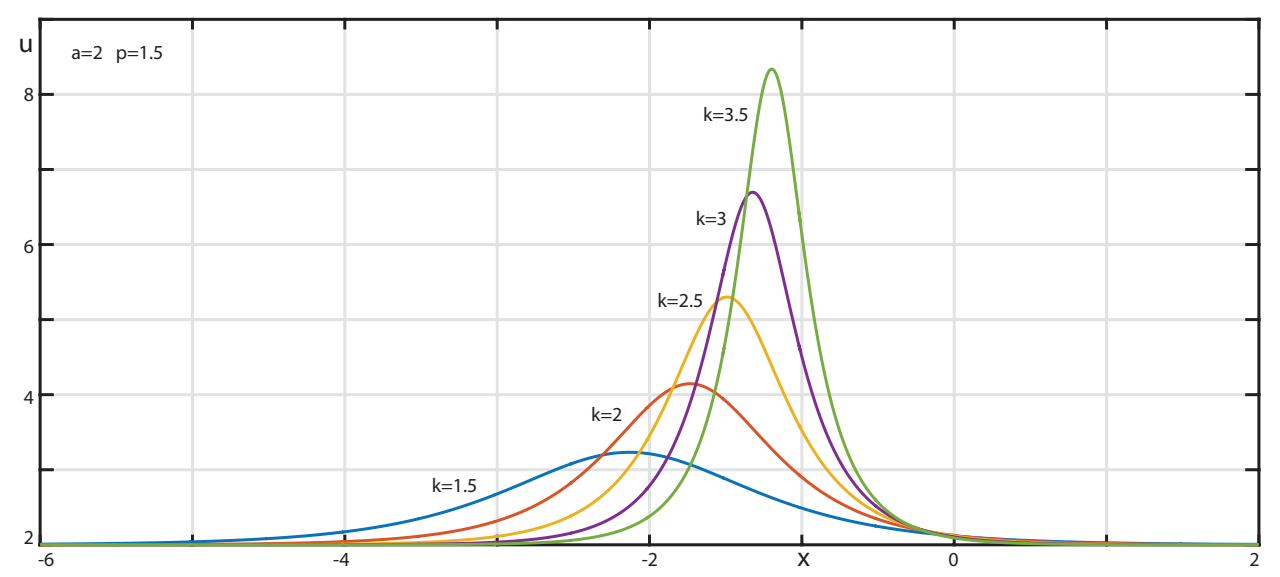

Fig. 9. The typical profiles of the soliton family (3.49) of the A- equation.

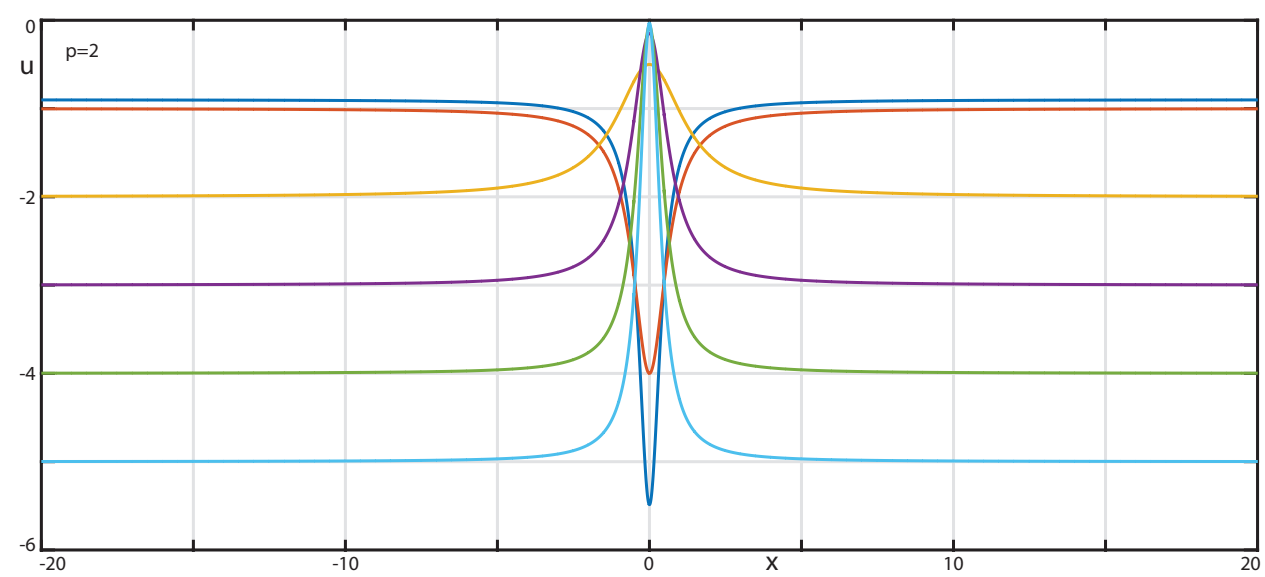

Fig. 10. The typical profiles of the algebraic solitons (3.50) of the A- equation. 
at the monoms $V_{1}^{n_{1}} V_{2}^{n_{2}}$ to zero, and those system will be compatible. Handling in this manner, after the simple algebra we have just

$$
u(x, t)=V_{1}(x, t)-V_{2}(x, t)
$$

with $C_{1}(x, t), C_{2}(x, t)$ and $S_{1}(x, t), S_{2}(x, t)$ such that

$$
C_{1}=S_{1}-3 S_{2}, \quad C_{2}=S_{2}-3 S_{1}, \quad S_{1}-S_{2} \pm 2 p=0 .
$$

In terms of the phase functions $\theta_{1}, \theta_{2}$ (3.52) give two evolution equations and the one constraint

$$
\begin{gathered}
\theta_{j, t}=\Theta_{j}\left(\theta_{1, x}, \theta_{1, x x}, \theta_{1, x x x} ; \theta_{2, x}, \theta_{2, x x}, \theta_{2, x x x} ; k, v ; p\right), \quad j=1,2, \\
G\left(\theta_{1, x}, \theta_{1, x x}, \theta_{1, x x x} ; \theta_{2, x}, \theta_{2, x x}, \theta_{2, x x x} ; k ; p\right)=0
\end{gathered}
$$

for the SF (3.51) of the form

$$
u(x, t)=U\left(T_{1}, T_{2} ; \theta_{1, x}, \theta_{1, x x}, \theta_{1, x x x} ; \theta_{2, x}, \theta_{2, x x}, \theta_{2, x x x} ; k\right) .
$$

In contrast to the analogous expressions (3.44)-(3.46), all the expressions (3.53)-(3.55) do not depend on $\theta_{1}, \theta_{2}$ explicitly, so that they appear to be invariant with respect to the change $\theta_{j}(x, t) \rightarrow$ $\theta_{j}(x, t)+\Delta_{j}(j=1,2)$. That is both kinks associated with $V_{1}, V_{2}$ in (3.51) can be positioned independently. Simultaneously, the SF says that they do not even interact with each other, their superposition is just linear. Any interactions take place only with the perturbation field. The related clear two-kink expression is

$$
\begin{aligned}
u_{\mathrm{k}}(x, t) & =\frac{k_{1}}{2} \tanh \left[\frac{k_{1}}{2}\left(x-v_{1} t+\Delta_{1}\right)\right]-\frac{k_{2}}{2} \tanh \left[\frac{k_{2}}{2}\left(x-v_{2} t+\Delta_{2}\right)\right], \\
k_{1,2} & >0, v_{1,2}, \Delta_{1,2}=\mathrm{const}
\end{aligned}
$$

with the special wave number and dispersion relations in view of (3.53), (3.54)

$$
k_{1}^{2}-k_{2}^{2} \mp 4 p=0, \quad v_{1}=\frac{1}{2}\left(3 k_{2}^{2}-k_{1}^{2}\right), \quad v_{2}=\frac{1}{2}\left(3 k_{1}^{2}-k_{2}^{2}\right) .
$$

For the degenerate case with $p=0$ (3.56), (3.57) is just two similar kinks moving with the identical velocities and identical asymptotics $u_{\mathrm{k}}(\infty, t)=0$. While for $p \neq 0$ this is already the nonstationary structure. Its kinks have the different velocities $\left|v_{1}-v_{2}\right|=8|p|$, and its asymptotics are also different $u_{\mathrm{k}}( \pm \infty, t)= \pm\left(k_{1}-k_{2}\right) / 2$.

The obtained results shows that for Eq. (3.37) together with the along kink solutions there exist both their strong bound states, the bell-shape solitons (3.48), and their absolutely noninteracting configurations (3.56) for the special choice of the wave numbers (3.57). And both complexes elastically interact with perturbations.

\section{The Linearization and Parametrization of the SFs and the Soliton Interactions Analysis}

The full SF analysis is not impossible and can gives interesting and important information [6]. However, as a rule, such SFs are huge enough. For our cases with two branches there is one more difficulty, the full SFs include two linked modulation functions. Fortunately, there are many characteristics of the soliton-perturbations interactions which can easily be obtained from the linearized 
SFs. Moreover, for the SFs generated by two branches there exists the simple procedure allowing one to introduce the only modulation function or to 'parameterize' such two functions SFs. This section is devoted to these procedures and to such analysis. Add here those linearized SFs are of their own rights as well. For instance, they can be applied also for describing week interactions [17], bounded soliton states $[23,24]$, stability $[21,22]$, etc. See in this connection also the works $[25,26]$.

\subsection{The MKdV equation}

We will consider the formulae for the soliton family (3.17) of the MKdV - equation (3.1) first, and then these formulae will be extended to other cases, (3.18) and (3.21). One will begin with the linearization of Eqs. (3.10), (3.11) to the function $\theta_{1}$ and $\theta_{2}$ and introduce the small parameter $\varepsilon$ for this in the following obvious manner

$$
\theta_{j}(x, t)=\varepsilon \varphi_{j}(x, t)+\mathrm{o}(\varepsilon), \quad j=1,2, \quad|\varepsilon| \ll 1 .
$$

Remaining the terms up to the first order with respect to $\varepsilon$, one has

$$
\begin{aligned}
& \varphi_{j, t}=-\varphi_{j, x x x}+6 a^{2} \varphi_{j, x}, \quad j=1,2, \\
& D_{1} \varphi_{1}=D_{2} \varphi_{2}, \quad D_{1,2}= \pm \frac{\partial}{\partial x}+2 a .
\end{aligned}
$$

First, we can assume $a \neq 0$ here, because this would lead to the singularity in the solutions (3.17). The special symmetrical structure of the constraint (4.3) allows one to turn easily to the only modulation function. Indeed, first of all notice that (4.3) is the inhomogeneous linear ordinary differential equation with respect to $\varphi_{1}$ or $\varphi_{2}$, and hence

$$
\varphi_{j}(x, t)=\varphi_{j, \mathrm{p}}(x, t)+\varphi_{j, \mathrm{~g}}(x, t), \quad j=1,2 .
$$

Where $\varphi_{j, \mathrm{p}}$ are some particular solutions, while $\varphi_{j, \mathrm{p}}$ are the general solutions of the related ODEs $D_{1} \varphi_{1}=0, D_{2} \varphi_{2}=0$. Since (4.3) can always be converted to the identity

$$
D_{1} D_{2} D_{\mathrm{L}} \varphi(x, t) \equiv D_{2} D_{1} D_{\mathrm{L}} \varphi(x, t)
$$

( $D_{\mathrm{L}}$ is any integro-differential operator), $\varphi_{1, \mathrm{p}}$ and $\varphi_{2, \mathrm{p}}$ can simultaneously be expressed through some auxiliary function $\varphi(x, t)$ choosing $\varphi_{1, \mathrm{p}}=D_{2} D_{\mathrm{L}} \varphi$ and $\varphi_{2, \mathrm{p}}=D_{1} D_{\mathrm{L}} \varphi$ such that $\left.\varphi_{1, \mathrm{p}}\right|_{\varphi=0}=$ $\left.\varphi_{1, \mathrm{p}}\right|_{\varphi=0}=0$. In doing so, $\varphi_{1, \mathrm{~g}}$ and $\varphi_{2, \mathrm{~g}}$ should be set to zero $\varphi_{1, \mathrm{~g}}=\varphi_{2, \mathrm{~g}}=0$, because according to our assumption at $\varphi=0$ only the clear soliton without any perturbations has to remain. So, for (4.3) the following simple parametrization takes place

$$
\varphi_{1,2}=2 a \varphi \mp \varphi_{x}, \quad \varphi_{t}=-\varphi_{x x x}+6 a^{2} \varphi_{x} .
$$

The situation with the related expression for $u$

$$
u(x, t)=u_{0}(x, t)+\varepsilon u_{1}(x, t)+\mathrm{o}(\varepsilon)
$$

for the SF (3.9) is more sophisticated. The trick here is to remain the modulated phase of the soliton as is. And the goal of this is not even to obtain the more compact and clear expression. On the strength of the asymptotics of $T_{1,2}\left(x_{1}, t_{1}, x_{2}, t_{2}\right)(2.39)$ corresponding to the infinite separation of the 
soliton and perturbation this makes the investigation of the interactions more straightforward. And last but not least, this provides the uniform convergence for such SFs. So, as a result, one has

$$
u_{0}=\frac{k}{2}\left(T_{1}-T_{2}\right)+a, u_{1}=k\left[a\left(T_{1}-T_{2}\right)+\frac{k}{2}\right] \varphi_{x}-\frac{k}{2}\left(T_{1}+T_{2}\right) \varphi_{x x}+\frac{\varphi_{x x x}}{2} .
$$

Let us now consider how the linearization can be used for analysis of the interactions of the solitons with linear waves and with other solitons. The function $\varphi$ with the asymptotics $\varphi( \pm \infty, t)=$ $\varphi_{ \pm \infty}=$ const corresponds to usual elastic soliton-perturbation interactions [6]. While the states of the soliton before and after the interactions distinguish just by its phases with the shift depending on the difference $\varphi_{+\infty}-\varphi_{-\infty}$, the expressions for the perturbations in view of $u_{1}$ in (4.6) with (2.39) are as follows

$$
u_{\mathrm{p}_{ \pm \infty}}(x, t)=\frac{k^{2}}{2} \varphi_{x} \mp k \varphi_{x x}+\frac{\varphi_{x x x}}{2},
$$

see the analogous example with the $\mathrm{MKdV}_{-}$kink (2.9)-(2.12). The simplest consequence from (4.5), (4.6) is the transfer function

$$
T_{-\infty}^{+\infty}(\kappa)=\frac{\sigma_{+\infty}\left(k_{\mathrm{p}}\right)}{\sigma_{-\infty}\left(k_{\mathrm{p}}\right)}=\frac{\kappa-2 \kappa^{2}+\kappa^{3}}{\kappa+2 \kappa^{2}+\kappa^{3}}, \quad \kappa=\frac{k_{\mathrm{p}}}{k}
$$

for the Fourier modes of small perturbations before and after interactions [11]

$$
u_{\mathrm{p}_{ \pm \infty}}(x, t)=\int_{-\infty i}^{+\infty i} \sigma_{ \pm \infty}\left(k_{\mathrm{p}}\right) \mathrm{e}^{\left[k_{\mathrm{p}} x+\omega\left(k_{\mathrm{p}}\right) t\right]} \mathrm{d} k_{\mathrm{p}} .
$$

Such a transfer function can also be used to determine the phase shifts of other solitons after the interactions. Indeed, the phase shift of the whole soliton is obviously identical to the phase shift of its exponential fronts, say, the left front, e.g. for (3.17) one has

$$
u_{\mathrm{s}_{1}}(x, t)=a+2 k^{2} \mathrm{e}^{\eta}+8 a k^{2} \mathrm{e}^{2 \eta}+\cdots, \quad \eta=k(x-v t+\Delta) .
$$

Here the constant term is inessential because is already included into $u_{0}$ (4.6), just the second exponential one is needed. So, this transfer function gives the soliton phase shift dependence on the wave numbers of both solitons taking part in the colliding

$$
\Delta_{\mathrm{p}}\left(k, k_{\mathrm{p}}\right)=-\frac{1}{k_{\mathrm{p}}} \ln T_{-\infty}^{+\infty}\left(\frac{k_{\mathrm{p}}}{k}\right) .
$$

As was demonstrated in the previous section, the real value exponential soliton (3.18) for the $\mathrm{MKdV}_{+}$is basically the imaginary version of the soliton (3.17) of the $\mathrm{MKdV}_{-}$and can be obtained from the latter via the simplest transformation $a \rightarrow i a, u \rightarrow i u$. For its perturbed version we should do the same also for the perturbation function, i.e. $\varphi \rightarrow i \varphi$. So, we will have the parametrization

$$
\varphi_{1,2}=-2 a \varphi \mp i \varphi_{x}, \quad \varphi_{t}=-\varphi_{x x x}-6 a^{2} \varphi_{x} .
$$

together with the expressions for the different components of the SF (4.5)

$$
u_{0}=-\frac{k}{2} i\left(T_{1}-T_{2}\right)+a, u_{1}=k\left[a i\left(T_{1}-T_{2}\right)+\frac{k}{2}\right] \varphi_{x}-\frac{k}{2}\left(T_{1}+T_{2}\right) \varphi_{x x}+\frac{\varphi_{x x x}}{2} .
$$

This case is more interesting than the previous one with the $\mathrm{MKdV}_{-}$solitons. In particular, here the functions $\varphi_{1,2}$ appear to be also complex, see (4.8). As a result, $T_{1}$ and $T_{2}$ may oscillate even for not 
oscillating $\varphi$, see (2.51), (2.52) for the ingredients in $u_{0}$ and $u_{1}$ (4.9), here

$$
\mu_{1}=k x-k\left(6 a^{2}+k^{2}\right) t+\Delta+\frac{\ln \left(4 a^{2}+k^{2}\right)}{2}-2 \varepsilon a k \varphi, \mu_{2}=\arg (2 a+k i)-\varepsilon k \varphi_{x} .
$$

All the above analysis of the SF is also suitable for this case in the whole volume.

Deriving all the above formulae, we assumed that $a \neq 0$ in (4.3). What will we have when $a=0$ however? First of all, in this case the $\mathrm{MKdV}_{-}$soliton (3.17) has the singularity on the real axis, so it is necessary to consider the case with the $\mathrm{MKdV}_{+}$(3.18). Let us return to Eqs. (4.2), (4.3). Now instead of (4.4) they will be

$$
\varphi_{j, t}=-\varphi_{j, x x x}(j=1,2), \quad \varphi_{1, x}+\varphi_{2, x}=0 .
$$

The second equation in (4.11) is very simple, and the parametrization with the new function $\varphi(x, t)$ is of the form

$$
\varphi_{1,2}=\mp i \varphi, \quad \varphi_{t}=-\varphi_{x x x} .
$$

So that for $u_{0}, u_{1}$ in (4.5) one has

$$
\begin{gathered}
u_{0}=-\frac{k}{2} i\left(T_{1}-T_{2}\right), \quad u_{1}=\frac{k^{2}}{2} \varphi-\frac{k}{2}\left(T_{1}+T_{2}\right) \varphi_{x}+\frac{\varphi_{x x}}{2}, \\
\mu_{1}=k x-k^{3} t+\Delta+\ln k, \quad \mu_{2}=\frac{\pi}{2}-\varepsilon k \varphi, \quad k>0 .
\end{gathered}
$$

In contrast to the analogous expression (4.9), here $u_{1}$ in (4.13) contains the function $\varphi$ itself. This means that for the small linear perturbations their interactions with the soliton do not result in the phase shift of the last one. Indeed, for the linear perturbation before and after the interaction one has from (4.13)

$$
u_{\mathrm{p}_{ \pm \infty}}(x, t)=\frac{k^{2}}{2} \varphi \mp k \varphi_{x}+\frac{\varphi_{x x}}{2} .
$$

For localized perturbations $u_{\mathrm{p}}(\infty, t)=0$ corresponding to asymptotics $\varphi( \pm \infty, t)=\varphi_{ \pm}=$const this immediately means $\varphi_{ \pm \infty}=0$, and as the consequence the absent of any soliton phase shift. Figure 11 corresponds to such an interaction, while Figure 12 to the interaction of the type considered previously (4.9). To be precise, both they depict the computer simulation for the $\mathrm{MKdV}_{+}$equation linearized on the soliton backgrounds $v_{0}(3.18)$

$$
v_{t}+6\left(v_{0}^{2} v\right)_{x}+v_{x x x}=0, \quad v=v(x, t)
$$

and in the coordinate system moving with the soliton. On the plots we clearly see the result of interaction for the solitons, namely the related first order Taylor correction to the unperturbed soliton in the second case and its absence in the first one.

Next, from (4.14) the transfer function is

$$
T_{-\infty}^{+\infty}(\kappa)=\frac{1-2 \kappa+\kappa^{2}}{1+2 \kappa+\kappa^{2}}, \quad \kappa=\frac{k_{\mathrm{p}}}{k}
$$

that also gives the formula for the interacting soliton phase shift analogous to (4.7).

In conclusion let us consider the case (3.12)-(3.14) corresponding to the algebraic soliton (3.21). It is the most simple one. Here the nonsingular solutions exist only for the $\mathrm{MKdV}_{+}$and $a \neq 0$. In the 


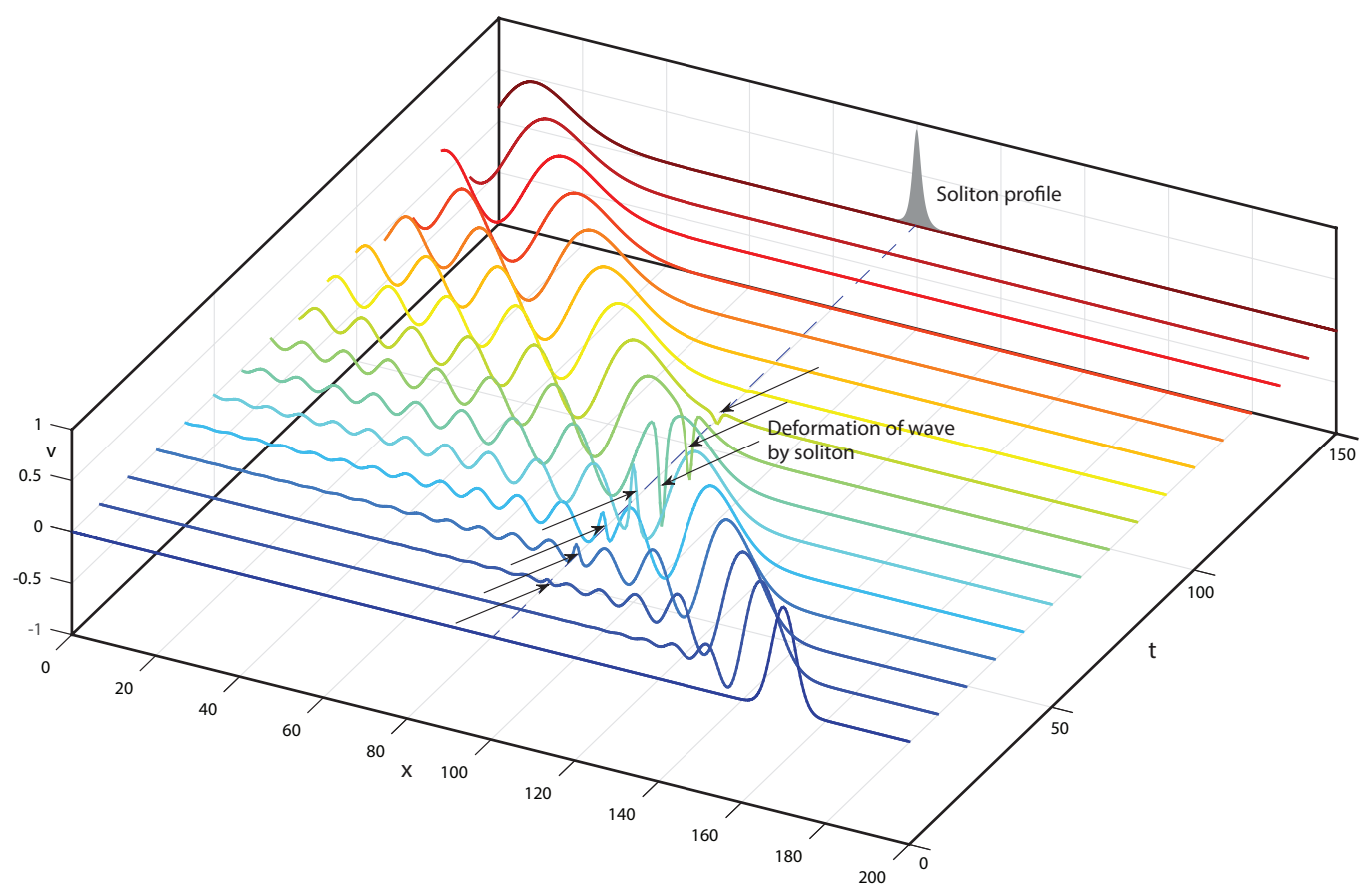

Fig. 11. The computer simulation of the first order perturbation equation (4.15) corresponding to the interaction with the $\mathrm{SF}$ (4.5), (4.13) of the $\mathrm{MKdV}_{+}$soliton (3.18) $(k=1, a=0)$ and some arbitrary linear wave package. The coordinate system is moving together with the soliton. The soliton phase shift is totally absent.

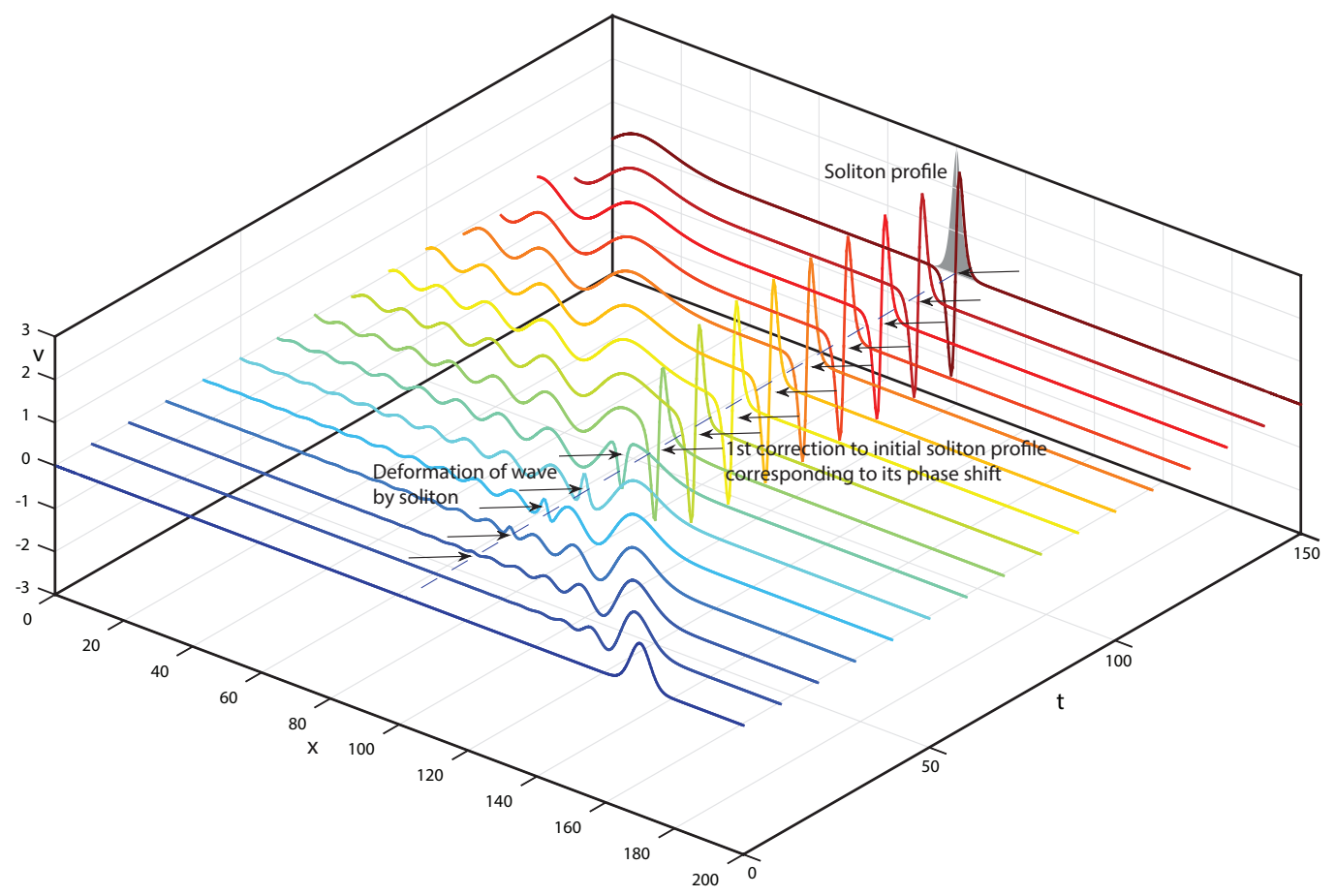

Fig. 12. The computer simulation of the first order perturbation equation (4.15) corresponding to the interaction with the $\mathrm{SF}$ (4.5), (4.9) of the $\mathrm{MKdV}_{+}$soliton (3.18) for $k=1$ and $a=0.5$ and some arbitrary linear wave package. The coordinate system is moving together with the soliton. The powerful soliton phase shift takes place after the interaction. 
view of the limiting relations (2.43)-(2.49) all the formulae sought can be trivially obtained from the analogous formulae (4.8)-(4.10). The parametrization (4.8) remain the same. While for the SF, from (4.9) one has

$$
u_{0}(x, t)=-i\left(P_{1}-P_{2}\right)+a, \quad u_{1}(x, t)=2 i a\left(P_{1}-P_{2}\right) \varphi_{x}-\left(P_{1}+P_{2}\right) \varphi_{x x}+\frac{\varphi_{x x x}}{2} .
$$

Both $u_{0}$ and $u_{1}$ are pure real in view of (2.51), (2.53), here

$$
\mu_{1}=x-6 a^{2} t+\Delta-2 \varepsilon a \varphi, \quad \mu_{2}=\frac{1}{2 a}-\varepsilon \varphi_{x}
$$

And because of the identical asymptotics of $P_{1,2}$ (2.41) the expressions being obtaining from $u_{1}$ (4.16) for the perturbation after and before the interaction are identical

$$
u_{\mathrm{p}_{ \pm \infty}}(x, t)=\frac{\varphi_{x x x}}{2}, \quad T_{-\infty}^{+\infty}=1 .
$$

So there is no effect of the soliton to it finally, the algebraic soliton is transparent. However, the soliton itself experiences the phase shift $2 \varepsilon a\left(\varphi_{+\infty}-\varphi_{-\infty}\right)$, see (4.17), as usually.

\subsection{The KK equation}

As was said and demonstrated above, all the relations (3.29), (3.30) to $\theta_{1}, \theta_{2}$ and the SF (3.31) itself for the KK equation (3.23) are huge enough. So, we are obliged to confine ourselves in our narrative by only the main algebra and results. Fortunately, both the algebra and the ideas basically remains the same as for the MKdV.

Instead of the original equations (3.29), (3.30) to the modulation functions $\theta_{1}$ and $\theta_{2}$ in the SF (3.31) the linearization procedure with (4.1) $\theta_{j}(x, t)=\varepsilon \varphi_{j}(x, t)+\mathrm{o}(\varepsilon)(j=1,2)$ gives two linear evolution equations

$$
\begin{aligned}
\varphi_{1, t}= & A_{1} \varphi_{1}+A_{2} \varphi_{1, x}+A_{4} \varphi_{1,2 x}+A_{6} \varphi_{1,3 x}+A_{8} \varphi_{1,4 x}-\frac{1}{2} \varphi_{1,5 x}-A_{1} \varphi_{2} \\
& +A_{3} \varphi_{2, x}+A_{5} \varphi_{2,2 x}+A_{7} \varphi_{2,3 x}-\frac{1}{2} \varphi_{2,5 x} \\
\varphi_{2, t}= & A_{1} \varphi_{1}+A_{3} \varphi_{1, x}-A_{5} \varphi_{1,2 x}+A_{7} \varphi_{1,3 x}-\frac{1}{2} \varphi_{1,5 x}-A_{1} \varphi_{2}+A_{2} \varphi_{2, x} \\
& -A_{4} \varphi_{2,2 x}+A_{6} \varphi_{2,3 x}-A_{8} \varphi_{2,4 x}-\frac{1}{2} \varphi_{2,5 x},
\end{aligned}
$$

where the coefficients $A_{j}$ are as follows

$$
\begin{gathered}
A_{1}=5 s\left(k^{6}+42 k^{4} a+504 k^{2} a^{2}+1728 a^{3}\right) /(3 k), \\
A_{2}=-9\left(k^{4}+22 k^{2} a+120 a^{2}\right) / 2, \quad A_{3}=-\left(k^{4}+102 k^{2} a+720 a^{2}\right) / 2, \\
A_{4}=14 s\left(k^{4}+12 k^{2} a+36 a^{2}\right) /(3 k), \quad A_{5}=2 s\left(5 k^{4}-12 k^{2} a-252 a^{2}\right) /(3 k), \\
A_{6}=-15 a, \quad A_{7}=3\left(k^{2}+a\right), \quad A_{8}=-2 s\left(k^{2}+6 a\right) / k,
\end{gathered}
$$

with the linear constraint (note $k^{2}+12 a \neq 0$ and $k^{2}+6 a \neq 0$ for the relevant cases)

$$
D_{1} \varphi_{1}=D_{2} \varphi_{2}, \quad D_{1,2}=k \frac{\partial^{3}}{\partial x^{3}} \mp 2 s\left(k^{2}+6 a\right) \frac{\partial^{2}}{\partial x^{2}}+k\left(k^{2}+12 a\right) \frac{\partial}{\partial x} .
$$




\section{A.A. Alexeyev / A multidimensional superposition principle: IV}

Then introducing into (4.18), (4.19) the parametrization with the new function $\varphi(x, t)$

$$
\varphi_{1,2}=\left(k^{2}+12 a\right) \varphi \pm 2 \frac{s}{k}\left(k^{2}+6 a\right) \varphi_{x}+\varphi_{2 x}, \varphi_{t}=-\varphi_{5 x}-30 a \varphi_{3 x}-180 a^{2} \varphi_{x}
$$

in view of the structure of the constraint (4.19), we arrive at the linearized SF

$$
\begin{aligned}
& u(x, t)=u_{0}(x, t)+\varepsilon u_{1}(x, t), \quad u_{0}=-\frac{k^{2}}{8}\left(T_{1}^{2}+T_{2}^{2}\right)+\frac{k^{2}}{4}+a, \\
& u_{1}=\left[\left(\frac{k^{2}}{3}+3 a\right)-\left(\frac{k^{2}}{4}+3 a\right)\left(T_{1}^{2}+T_{2}^{2}\right)\right] k^{2} \varphi_{x}+\left[\left(\frac{k^{2}}{4}+3 a\right) \times\right. \\
& \left.\left(T_{1}+T_{2}\right)-s\left(\frac{k^{2}}{2}+3 a\right)\left(T_{1}^{2}-T_{2}^{2}\right)\right] k \varphi_{2 x}+\left[s\left(\frac{k^{2}}{2}+3 a\right)\left(T_{1}-T_{2}\right)-\right. \\
& \left.\frac{k^{2}}{4}\left(T_{1}^{2}+T_{2}^{2}\right)-\left(\frac{k^{2}}{6}+3 a\right)\right] \varphi_{3 x}+\left(T_{1}+T_{2}\right) \frac{k}{4} \varphi_{4 x}-\frac{1}{6} \varphi_{5 x} .
\end{aligned}
$$

Having in the hands the formulae (4.21), we can study the interactions features. The limited values of the functions $T_{1,2}(2.39)$ are directly associated with the asymptotical states of the perturbations before and after the interaction (in the infinity in the left or in the right of the soliton). As a result, for the perturbation before and after the interaction the expression $u_{1}$ in (4.21) gives

$$
u_{\mathrm{p}_{ \pm \infty}}(x, t)=-\frac{k^{3}}{6}\left(k^{2}+18 a\right) \varphi_{x} \pm \frac{k^{2}}{2}\left(k^{2}+12 a\right) \varphi_{2 x}-\frac{k}{3}\left(2 k^{2}+9 a\right) \varphi_{3 x} \pm \frac{k^{2}}{2} \varphi_{4 x}-\frac{k}{6} \varphi_{5 x} .
$$

As a consequence, for the transfer function $T_{-\infty}^{+\infty}$ one obtains the following expressions

$$
T_{-\infty}^{+\infty}(\kappa)=\frac{(18 \rho+1)-3 \kappa(12 \rho+1)+2 \kappa^{2}(9 \rho+2)-3 \kappa^{3}+\kappa^{4}}{(18 \rho+1)+3 \kappa(12 \rho+1)+2 \kappa^{2}(9 \rho+2)+3 \kappa^{3}+\kappa^{4}}, \quad \kappa=\frac{k_{\mathrm{p}}}{k}, \rho=\frac{a}{k^{2}} .
$$

The formulae (4.20)-(4.23) themselves are right both for the soliton (3.33) and for (3.34) without any changes. However, for the latter, the parameters $s$ will be purer imaginary. The expressions for $u_{0}, u_{1}$ (4.21) will be real in any case (see (2.51), (2.52) for their ingredients), but $\varphi_{1,2}$ in (4.20) appear to be complex. So, the SF will include sine and cosine functions. For $\mu_{1}, \mu_{2}$ we will have

$$
\begin{gathered}
\mu_{1}=k x-\left(k^{4}+30 a k^{2}+180 a^{2}\right) k t+\frac{\ln \left(1+\hat{s}^{2}\right)}{2}+\varepsilon\left[\left(k^{2}+12 a\right) \varphi+\varphi_{x x}\right], \\
\mu_{2}=\arg (1+\hat{s} i)+2 \varepsilon \frac{\hat{s}}{k}\left(k^{2}+6 a\right) \varphi_{x} .
\end{gathered}
$$

For the nonsingular algebraic soliton (3.36) $(a<0)$ all analogous formulae can be obtained from the above formulae for the exponential solitons as the limit at $k \rightarrow 0$ (see (2.48), (2.50)). Instead of (4.20), the following expressions take place, see (3.35),

$$
\varphi_{1,2}=12 a \varphi \pm 12 i \hat{d} a \varphi_{x}+\varphi_{2 x}, \quad \varphi_{t}=-\varphi_{5 x}-30 a \varphi_{3 x}-180 a^{2} \varphi_{x}, \quad \hat{d}^{2}=-\frac{1}{8 a} .
$$

While (4.21) gives

$$
\begin{aligned}
& u(x, t)=u_{0}(x, t)+\varepsilon u_{1}(x, t)+\mathrm{o}(\varepsilon), \quad u_{0}=-\frac{1}{2}\left(P_{1}^{2}+P_{2}^{2}\right)+a, \\
& u_{1}=-12 a\left(P_{1}^{2}+P_{2}^{2}\right) \varphi_{x}+6 a\left[\left(P_{1}+P_{2}\right)-2 i \hat{d}\left(P_{1}^{2}-P_{2}^{2}\right)\right] \varphi_{2 x}+ \\
& {\left[6 i \hat{d} a\left(P_{1}-P_{2}\right)-\left(P_{1}^{2}+P_{2}^{2}\right)-3 a\right] \varphi_{3 x}+\frac{\left(P_{1}+P_{2}\right)}{2} \varphi_{4 x}-\frac{1}{6} \varphi_{5 x}}
\end{aligned}
$$


where, see (2.51),

$$
\mu_{1}=x-180 a^{2} t+\varepsilon\left(12 a \varphi+\varphi_{x x}\right), \quad \mu_{2}=\hat{d}+12 \varepsilon \hat{d} a \varphi_{x} .
$$

As well as the soliton (3.36) itself, $u_{0}, u_{1}$ (4.24) in the SF are real in view of (2.53). Also, the expressions

$$
u_{\mathrm{p}_{ \pm \infty}}(x, t)=-3 a \varphi_{3 x}-\frac{\varphi_{5 x}}{6}, \quad T_{-\infty}^{+\infty}=1
$$

for the perturbations take place.

\subsection{The $A_{ \pm}$equations}

The algebra and formulaes of the linearization of the $\mathrm{A}_{ \pm}$equations (3.37) principally remain the same as in the cases with the MKdV and KK equations. So, only their main points and final results are given here.

\subsubsection{The bell-shape exponential and algebraic soliton cases}

The transition to the linearized versions of the determining equations (3.44), (3.45) via the substitution (4.1) $\theta_{j}(x, t)=\varepsilon \varphi_{j}(x, t)+o(\varepsilon)(j=1,2)$ gives the pair of the linear evolution equations

$$
\begin{gathered}
\varphi_{1, t}=A_{1} \varphi_{1}+A_{2} \varphi_{1, x}+A_{3} \varphi_{1, x x}-A_{1} \varphi_{2}+A_{4} \varphi_{2, x}+A_{5} \varphi_{2, x x}+\varphi_{1, x x x}+3 \varphi_{2, x x x}, \\
\varphi_{2, t}=A_{1} \varphi_{1}+A_{4} \varphi_{1, x}-A_{5} \varphi_{1, x x}-A_{1} \varphi_{2}+A_{2} \varphi_{2, x}-A_{3} \varphi_{2, x x}+3 \varphi_{1, x x x}+\varphi_{2, x x x}
\end{gathered}
$$

where

$$
\begin{gathered}
A_{0}=2 a^{5}\left(k^{2} a^{2}-3 p^{2}-a^{4}\right), \\
A_{1}=3\left(k^{4} a^{8}-k^{4} p^{2} a^{4}+2 k^{2} p^{4} a^{2}-2 k^{2} a^{10}-p^{6}+3 p^{4} a^{4}-3 p^{2} a^{8}+a^{12}\right) / A_{0}, \\
A_{2}=a^{3}\left(2 k^{4} a^{4}-5 k^{2} p^{2} a^{2}-7 k^{2} a^{6}+5 p^{4}+6 p^{2} a^{4}+15 a^{8}\right) / A_{0}, \\
A_{3}=3 a^{2}\left(k^{4} a^{4}-2 k^{2} p^{2} a^{2}-4 k^{2} a^{6}+p^{4}+4 p^{2} a^{4}+3 a^{8}\right) / A_{0}, \\
A_{4}=3 a^{3}\left(2 k^{4} a^{4}-5 k^{2} p^{2} a^{2}-3 k^{2} a^{6}+5 p^{4}-6 p^{2} a^{4}+a^{8}\right) / A_{0}, \\
A_{5}=3 a^{2}\left(-k^{4} a^{4}+2 k^{2} p^{2} a^{2}-p^{4}+8 p^{2} a^{4}+a^{8}\right) / A_{0}
\end{gathered}
$$

with the linkage between $\varphi_{1}$ and $\varphi_{2}$

$$
D_{1} \varphi_{1}=D_{2} \varphi_{2}, \quad D_{1,2}=a^{2} \frac{\partial^{3}}{\partial x^{3}} \pm 2 a^{3} \frac{\partial^{2}}{\partial x^{2}}+\left(a^{4}-p^{2}\right) \frac{\partial}{\partial x} .
$$

Eqs. (4.25) allow the parametrization with the new function $\varphi(x, t)$

$$
\varphi_{1,2}=\left(a^{4}-p^{2}\right) \varphi \mp 2 a^{3} \varphi_{x}+a^{2} \varphi_{x x}, \quad \varphi_{t}=2 \varphi_{x x x}-3 \frac{\left(p^{2}+a^{4}\right)}{a^{2}} \varphi_{x} .
$$

In terms of this new function the linearized SF will be of the form

$$
\begin{aligned}
& u(x, t)=u_{0}(x, t)+\varepsilon u_{1}(x, t)+\mathrm{o}(\varepsilon), \\
& u_{0}=\frac{k}{2}\left(T_{1}-T_{2}\right)+a, \quad u_{1}=\left[\left(T_{1}-T_{2}\right)\left(a^{4}-p^{2}\right)+2 k a^{3}\right] k \frac{\varphi_{x}}{2} \\
& \quad-a^{3}\left(T_{1}+T_{2}\right) k \varphi_{x x}+\frac{a^{2}}{2}\left[2 a+k\left(T_{1}-T_{2}\right)\right] \varphi_{x x x} .
\end{aligned}
$$


The related expressions for small, linear perturbations from (4.27) are as follows

$$
u_{\mathrm{p}_{ \pm \infty}}(x, t)=a^{3}\left(k^{2} \varphi_{x} \mp 2 k \varphi_{x x}+\varphi_{x x x}\right), \quad T_{-\infty}^{+\infty}(\kappa)=\frac{\kappa-2 \kappa^{2}+\kappa^{3}}{\kappa+2 \kappa^{2}+\kappa^{3}}, \quad \kappa=\frac{k_{\mathrm{p}}}{k} .
$$

For the exponential soliton (3.49) of the $\mathrm{A}_{-}$equation it is enough to use the change $a \rightarrow i a$ and $u \rightarrow i u$. Instead of the formulae (4.26) and (4.27), one will have

$$
\varphi_{1,2}=\left(a^{4}-p^{2}\right) \varphi \pm 2 i a^{3} \varphi_{x}-a^{2} \varphi_{x x}, \quad \varphi_{t}=2 \varphi_{x x x}+3 \frac{\left(p^{2}+a^{4}\right)}{a^{2}} \varphi_{x}
$$

and respectively

$$
\begin{aligned}
u_{0} & =-\frac{k}{2} i\left(T_{1}-T_{2}\right)+a, \quad u_{1}=\left[-i\left(T_{1}-T_{2}\right)\left(a^{4}-p^{2}\right)-2 k a^{3}\right] k \frac{\varphi_{x}}{2} \\
& +a^{3}\left(T_{1}+T_{2}\right) k \varphi_{x x}+\frac{a^{2}}{2}\left[-2 a+i k\left(T_{1}-T_{2}\right)\right] \varphi_{x x x}
\end{aligned}
$$

As seen from (4.29), $\varphi_{1,2}$ are complex here, so it is needed to take into account the relations (2.51) and (2.52) with

$$
\begin{aligned}
& \mu_{1}=k x+k\left(2 k^{2}+3 \frac{p^{2}+a^{4}}{a^{2}}\right) t+\Delta+\frac{\ln \left[\left(a^{4}-a^{2} k^{2}-p^{2}\right)^{2}+a^{2} k^{2}\right]}{2}+ \\
& \varepsilon k\left[\left(a^{4}-p^{2}\right) \varphi-a^{2} \varphi_{x x}\right], \quad \mu_{2}=\arg \left(a^{4}-a^{2} k^{2}-p^{2}+i a k\right)+2 \varepsilon k a^{3} \varphi_{x} .
\end{aligned}
$$

The expressions for $u_{\mathrm{p}_{ \pm \infty}}$ and $T_{-\infty}^{+\infty}$ (4.28) save their form. In its turn for the algebraic soliton (3.50) of the $\mathrm{A}_{-}$equation their analogs can be obtained in the same manner as before

$$
\begin{aligned}
& u_{0}=-i\left(P_{1}-P_{2}\right)+a \\
& u_{1}=-i\left(P_{1}-P_{2}\right)\left(a^{4}-p^{2}\right) \varphi_{x}+2 a^{3}\left(P_{1}+P_{2}\right) \varphi_{x x}+a^{2}\left[i\left(P_{1}-P_{2}\right)-a\right] \varphi_{x x x}
\end{aligned}
$$

and

$$
\begin{gathered}
\mu_{1}=x+3 \frac{\left(p^{2}+a^{4}\right)}{a^{2}} t+\Delta+\varepsilon k\left[\left(a^{4}-p^{2}\right) \varphi-a^{2} \varphi_{x x}\right], \quad \mu_{2}=\frac{-2 a^{3}}{p^{2}-a^{4}}+2 \varepsilon k a^{3} \varphi_{x} \\
u_{\mathrm{p}_{ \pm \infty}}(x, t)=a^{3} \varphi_{x x x}, \quad T_{-\infty}^{+\infty}=1
\end{gathered}
$$

i.e. as the degenerations at $k \rightarrow 0$.

As the comment note that at $a^{4}-p^{2}=0$ for the SFs (4.27), (4.30), (4.31) of all three soliton families there take place their degeneration. Indeed, in this case all formulae (4.26), (4.27) and (4.29), (4.30), (4.31) stop to depend on $\varphi$ explicitly, only its derivatives remain. First, this means that in this case there are only three modulation parameters $\left\{\varphi_{x}, \varphi_{x x}, \varphi_{x x x}\right\}$ instead fore. Second, we here have the same situation as with the MKdV degeneration (4.12), (4.13) (it is possibly to pass to another function $\varphi_{x} \rightarrow \varphi$ in the above formulae for the clearness), when any soliton phase shift after the interactions is absent. This effect takes place for both exponential soliton families (3.48) and (3.49), in contrast to the MKdV case with the family (3.18) only.

\subsubsection{The configuration from the two noninteracting kinks}

From the viewpoint of the formulae the case of the noninteracting kinks (3.56) in contrast to the alone bell-shaped soliton case has the only deference, namely the additional arbitrary constant $\Delta_{1,2}$ 
in the kink phases (3.56), that makes them independent on each other. The system of the linearized determining equations (3.53) after the change $\theta_{1,2} \rightarrow \Delta_{1,2}+\varepsilon \varphi_{1,2}+\mathrm{o}(\varepsilon)$, are fully similar and is

$$
\begin{aligned}
\varphi_{1, t} & =-\varphi_{1, x x x}+\frac{3}{2}\left(k_{1}^{2}-k_{2}^{2}\right) \varphi_{1, x}+3 \varphi_{2, x x x}-3 k_{2}^{2} \varphi_{2, x}, \\
\varphi_{2, t} & =3 \varphi_{1, x x x}-3 k_{1}^{2} \varphi_{1, x}-\varphi_{2, x x x}+\frac{3}{2}\left(k_{2}^{2}-k_{1}^{2}\right) \varphi_{2, x}, \quad \varphi_{1,2}=\varphi_{1,2}(x, t)
\end{aligned}
$$

with the constraint (3.54)

$$
D_{1} \varphi_{1}=D_{2} \varphi_{2}, \quad D_{1}=\frac{\partial^{3}}{\partial x^{3}}-k_{1}^{2} \frac{\partial}{\partial x}, \quad D_{2}=\frac{\partial^{3}}{\partial x^{3}}-k_{2}^{2} \frac{\partial}{\partial x} .
$$

After the parametrization of (4.32) in view of (4.33) with the function $\varphi(x, t)$

$$
\varphi_{1}=k_{2}^{2} \varphi-\varphi_{x x}, \quad \varphi_{2}=k_{1}^{2} \varphi-\varphi_{x x}, \quad \varphi_{t}=2 \varphi_{x x x}-\frac{3}{2}\left(k_{1}^{2}+k_{2}^{2}\right) \varphi_{x}
$$

the linearized SF (3.55) will be

$$
\begin{aligned}
& u(x, t)=u_{0}(x, t)+\varepsilon u_{1}(x, t)+o(\varepsilon), \quad u_{0}(x, t)=\frac{k_{1}}{2} T_{1}-\frac{k_{2}}{2} T_{2}, \\
& u_{1}(x, t)=\frac{k_{1}}{2}\left(k_{2}^{2} \varphi_{x}-\varphi_{x x x}\right) T_{1}-\frac{k_{2}}{2}\left(k_{1}^{2} \varphi_{x}-\varphi_{x x x}\right) T_{2}+\frac{\left(k_{1}^{2}-k_{2}^{2}\right)}{2} \varphi_{x x}
\end{aligned}
$$

with the expressions for the perturbation asymptotics from $u_{1}$ in (4.34)

$$
u_{\mathrm{p}_{ \pm \infty}}(x, t)=\left(\frac{k_{1}-k_{2}}{2}\right)\left[\mp k_{1} k_{2} \varphi_{x}+\left(k_{1}+k_{2}\right) \varphi_{x x} \mp \varphi_{x x x}\right] .
$$

Notice that the transfer function from (4.35) for these two-kink configuration

$$
T_{-\infty}^{+\infty}\left(\kappa_{1}, \kappa_{2}\right)=-\left(\frac{1-\kappa_{1}}{1+\kappa_{1}}\right)\left(\frac{1-\kappa_{2}}{1+\kappa_{2}}\right), \quad \kappa_{1}=\frac{k_{\mathrm{p}}}{k_{1}}, \kappa_{2}=\frac{k_{\mathrm{p}}}{k_{2}}
$$

basically is the composition of the transfer functions of the separate kinks.

\section{Conclusion}

The paper develops further the general theory of solitons and soliton interactions proposed by the author [3]- [11]. Theoretically, the article generalises the direct technique for constructing SFs [10]. And as its applications, the SFs for the MKdV, KK and $\mathrm{A}_{\mp}$ equations are considered. The results have been obtained for them concern both the fuller and deeper description of the known soliton solutions and the principally new results concerning their superposition properties and their interactions with other waves. The main points can be formulated in the following manner:

1. The above technique is generalized to the case of multibranches with constraints.

2. The formulae for the limit passage from the SFs for exponential solitons to algebraic ones are introduced.

3. The proposed generalization is used to derive the SFs for the solitons of the MKdV, KK and $\mathrm{A}_{\mp}$ nonlinear PDEs in the cases when one branch technique does not work. 
4. For all three equations the SFs for the bell-shape exponential and algebraic solitons are derived. It is shown these solitons can be considered as the strong bound states of two identical kinks (MKdV, $\mathrm{A}_{ \pm}$) or two simpler bell-shape solitons $(\mathrm{KK})$. For the $\mathrm{A}_{ \pm}$the two noninteracting kinks configuration is also found and studied.

5. Aside from the full SFs, their much more simple linearized versions are also investigated, and the parametrization procedure is introduced into the approach.

6. The linearized SFs are used for investigation of some interaction properties of the above solitons.

7. Based on the structure of the SFs constructed, the possible difference in the interactions (smooth and oscillating character) are indicated for the various SFs.

8. Based on the form of the SFs, the degenerated cases (i.e. with no soliton phase shift) are indicated for the $\mathrm{MKdV}$ and $\mathrm{A}_{\mp}$ and confirmed by the computer simulation.

9. On the example with the KK equation solitons the common viewpoint about the uselessness of the use of identical branches is disproved.

\section{Acknowledgements}

The author is grateful to Professor T Wolf for his assistance and together with Computer Canada and SHARCNET for the afforded computer facilities.

\section{References}

[1] M. J. Ablowitz, A. Ramani and H. Segur, Nonlinear evolution equations of Painlevé type, Lett. Nuovo Cim. 23 (1978) 333-338.

[2] M. J. Ablowitz and H. Segur, Solitons and the Inverse Scattering Transform (SIAM, 1981).

[3] A. A. Alexeyev, Superposition principles for nonlinear partial differential equations in $(1+1)$ dimension, Abstract of Short Communications and Poster Sessions, ICM 1998 Int. Cong. of Mathematicians, Berlin, August 18-27 (Rosenheim: Geronimo GmbH, 1998) 227.

[4] A. A. Alexeyev, A multidimensional superposition principle: classical solitons I, Preprint (Karlsruhe University, Evolve-L Archive, 1999) 99-00010.

[5] A. A. Alexeyev, A multidimensional superposition and singular manifold expansions, Abstracts, 3rd European Congress In Mathematics, Barcelona, July 10-14, 2000 electronically/CD.

[6] A. A. Alexeyev, A multidimensional superposition principle: classical solitons I, Phys. Lett. A 278 (2001) 198-208.

[7] A. A. Alexeyev, Multidimensional superposition principle and invariant manifolds of soliton type, Abstracts, Workshop Computer Algebra in Applications to Integrable Systems November 16-17, 2001 (Cambridge, Isaac Newton Institute for Mathematical Sciences) electronically.

[8] A. A. Alexeyev, A multidimensional superposition principle: classical solitons II, J. Phys. A: Math. Gen. 36 (2003) 9843-9864.

[9] A. A. Alexeyev, A multidimensional superposition principle and waves switching in integrable and nonintegrable soliton models, J. Phys. A: Math. Gen. 37 (2004) L627-L634.

[10] A. A. Alexeyev, A multidimensional superposition principle: classical solitons III, Phys. Lett. A 335 (2005) 197-206.

[11] A. A. Alexeyev, A multidimensional superposition principle: numerical simulation and analysis of soliton invariant manifolds I, J. Nonl. Math. Phys. 14 (2007) 188-229.

[12] A. A. Alexeyev, Nonlinear waves in a fluid with ultra small bubbles, Abstracts of Papers, XXXII Conf. of the PhM and NS Faculty, Part 2 (Moscow: PFU Publishing, 1996), 53-54.

[13] A. A. Alexeyev, Classical and non-classical interactions of kinks in some bubbly mediums, J. Phys. A: Math. Gen. 32 (1999) 4419-4432. 
[14] A. A. Alexeyev, Some notes on the singular manifold method: several manifolds and constraints, $J$. Phys. A: Math. Gen. 33 (2000) 1873-1894.

[15] S. C. Anco, N. T. Ngatat and M. Willoughby, Interaction properties of complex modified Kortewegde Vries (mKdV) solitons, Physica D 240 (2011) 1378-1394.

[16] R. Conte, Invariant Painlevé analysis of partial differential equations, Phys. Lett. A 140 (1989) 383-390.

[17] B. A. Dubrovin and S. P. Novikov, Hydrodynamics of weakly deformed soliton lattices. Differential geometry and Hamiltonian theory, Russ. Math. Surv. 44 (1989) 35-124.

[18] P. G. Estévez et al, Modified singular manifold expansion: application to the Boussinesq and MikhailovShabat systems, J. Phys. A: Math. Gen. 26 (1993) 1915-1925.

[19] J. D. Gibbon et al, Lax pairs Bäcklund transformations and special solutions for ordinary differential equations, Nonlinearity 1 (1988) 481-490.

[20] A. Heck, A birds-eye view of Gröbner bases, Nucl. Instrum. Methods A 389 (1997) 16-21.

[21] A. N. W. Hone and S. Lafortune, Stability of stationary solutions for nonintegrable peakon equations, Physica D 268 (2014) 28-36.

[22] Ch. K. R. T. Jones et al, On the stability analysis of periodic sine-Gordon traveling waves, Physica D 251 (2013) 63-74.

[23] T. Kawahara and M. Takaoka, Chaotic motions in an oscillatory soliton lattice, J. Phys. Soc. Japan 57 (1988) 3714-3732.

[24] T. Kawahara and S. Toh, Pulse interactions in an unstable dissipative-dispersive nonlinear system, Phys. Fluids 31 (1988) 2103-2111.

[25] A. P. Koller, Z. Hwang and M. Olshanii, Emergence of reflectionless scattering from linearizations of integrable PDEs around solitons, J. Phys. A: Math. Theor. 48 (2015) 075203.

[26] W. X. Ma and B. Fuchssteiner, Integrable theory of the perturbation equations, Chaos Soliton. Fract. 7 (1996) 1227-1250.

[27] E. L. Mansfield, Differential Gröbner bases, PhD Thesis (University of Sydney, 1992).

[28] M. Musette, R. Conte, The two-singular-manifold method: I. Modified Korteweg-de Vries and sineGordon equations, J. Phys. A: Math. Gen. 27 (1994) 3895-3913.

[29] A. Parker, On soliton solutions of the Kaup-Kupershmidt equation. I. Direct bilinearisation and solitary wave, Physica D 137 (2000) 25-33.

[30] A. Parker, On soliton solutions of the Kaup-Kupershmidt equation. II. Anomalous $N$-soliton solutions, Physica D 137 (2000) 34-48.

[31] J. Weiss, M. Tabor and G. Carneval, The Painlevé property for partial differential equations, J. Math. Phys. 24 (1983) 522-526.

[32] A. D. Wittkopf and G. J. Reid, The "Rif" package and documentation, Maple 6 and higher (2000).

[33] T. Wolf and A. Brand, The Computer Algebra Package CRACK for Investigating PDEs, REDUCE User's Manual, Version 3.7, A. C. Hearn and J. P. Fitch (Konrad-Zuze-Zentrum, 1999). 